\title{
OPEN GFAP splice variants fine-tune glioma cell invasion and tumour dynamics by modulating migration persistence
}

\author{
Rebeca Uceda-Castro ${ }^{1,4}$, Jessy V. van Asperen ${ }^{2,4}$, Claire Vennin ${ }^{1}$, Jacqueline A. Sluijs ${ }^{2}$, \\ Emma J. van Bodegraven ${ }^{2}$, Andreia S. Margarido ${ }^{1}$, Pierre A. J. Robe ${ }^{3}$, \\ Jacco van Rheenen ${ }^{1,5 \bowtie} \&$ Elly M. Hol ${ }^{2,5}$
}

Glioma is the most common form of malignant primary brain tumours in adults. Their highly invasive nature makes the disease incurable to date, emphasizing the importance of better understanding the mechanisms driving glioma invasion. Glial fibrillary acidic protein (GFAP) is an intermediate filament protein that is characteristic for astrocyte- and neural stem cell-derived gliomas. Glioma malignancy is associated with changes in GFAP alternative splicing, as the canonical isoform GFAP $\alpha$ is downregulated in higher-grade tumours, leading to increased dominance of the GFAP $\delta$ isoform in the network. In this study, we used intravital imaging and an ex vivo brain slice invasion model. We show that the GFAP $\delta$ and GFAP $\alpha$ isoforms differentially regulate the tumour dynamics of glioma cells. Depletion of either isoform increases the migratory capacity of glioma cells. Remarkably, GFAP $\delta$-depleted cells migrate randomly through the brain tissue, whereas GFAP $\alpha$-depleted cells show a directionally persistent invasion into the brain parenchyma. This study shows that distinct compositions of the GFAPnetwork lead to specific migratory dynamics and behaviours of gliomas.

Glioblastoma multiforme (GBM, grade IV glioma) is the most common and most aggressive tumour of the central nervous system, with an incidence of 3 per 100,000 people and a crude median survival of 9 months after diagnosis ${ }^{1}$. GBM is currently incurable and this is for a large part due to the highly invasive nature of glioma cells $^{2-4}$. Standard-of-care treatment for GBM consists of surgical tumour resection, followed by chemo- and radiotherapy, but fails to fully eradicate highly invasive glioma cells. As a consequence, patients often relapse after treatment and the tumour rapidly re-grows.

The intermediate filament (IF) protein glial fibrillary acid protein (GFAP) is a signature type III IF protein of glioma cells that has been implicated in tumour migration ${ }^{5-7}$. The role of IFs in glioma invasion and migration has only gained attention recently ${ }^{8}$. With over 70 genes encoding different IF proteins, the IF family is one of the largest human gene families and IF expression patterns are highly cell- and tissue type-specific ${ }^{9}$. Changes in the composition of the IFnetwork are associated with alterations in malignancy. For example, during the epithelialto-mesenchymal (EMT) transition, a process linked to increased cellular invasiveness and cancer progression ${ }^{10}$, the IFnetwork of cancer cells with an epithelial origin changes from a keratin-dominant to a vimentin-dominant network $^{11-13}$. In addition, breast cancer invasion is linked to changes in the IFnetwork, with a switch from keratin 8 to keratin 14 expression in invasive cells ${ }^{14}$. GFAP is an IF protein that is classically used to identify malignancies of glial origin, such as astrocytomas and glioblastomas ${ }^{15}$. In addition to GFAP, gliomas can heterogeneously express a combination of IFs including vimentin, synemin, and nestin ${ }^{16}$, which are located within the same filament in the cell ${ }^{17}$. GFAP is differentially spliced, and GFAP $\alpha$ and GFAP $\delta$ are the two isoforms that are most highly expressed and best studied. The GFAP $\delta$ isoform results from alternative splicing with a 3' polyadenylation event, where the last two exons 8 and 9 of GFAPa are replaced by exon 7 a, leading to an alternative 42 amino acid C-terminal tail ${ }^{18,19}$. The two isoforms have different assembly properties ${ }^{20}$, protein interactions ${ }^{19,21}$, and

\footnotetext{
${ }^{1}$ Division of Molecular Pathology, Oncode Institute, The Netherlands Cancer Institute, Amsterdam, The Netherlands. ${ }^{2}$ Department of Translational Neuroscience, University Medical Center Utrecht Brain Center, Utrecht University, Utrecht, The Netherlands. ${ }^{3}$ Department of Neurology and Neurosurgery, University Medical Center Utrecht Brain Center, University Utrecht, Utrecht, The Netherlands. ${ }^{4}$ These authors contributed equally: Rebeca Uceda-Castro and Jessy V. van Asperen. ${ }^{5}$ These authors jointly supervised this work: Jacco van Rheenen and Elly M. Hol. ${ }^{\circledR}$ email: j.v.rheenen@nki.nl; e.m.hol-2@umcutrecht.nl
} 
differ in their expression patterns, with GFAPa predominantly expressed in mature astrocytes and GFAP $\delta$ in the neurogenic niches of the human brain 22,23 .

In previous studies, we and others have shown that glioma malignancy is associated with alterations in GFAP splice isoform levels ${ }^{6,24-29}$. As such, RNA sequencing analysis of the cancer genome atlas (TCGA) database showed that increasing glioma malignancy grades are associated with a lower overall expression of GFAP and a shift towards higher levels of the alternative splice variant GFAP $\delta$ relative to GFAP $\alpha^{6}$. Increasing the GFAP $\delta / \alpha$ ratio in vitro leads to an upregulation of genes encoding proteins that are involved in the interactions between cells and the extracellular matrix (ECM) such as laminins, integrins, and matrix metalloproteinase 2 (MMP$2)^{5-7,20}$. Besides, immunohistochemical analysis of glioma tissue samples linked GFAP $\delta$ expression to an altered cellular morphology ${ }^{26,28}$ and to more invasive tumours based on neuroimaging ${ }^{27}$. Although these observations are suggestive for changed glioma cell behaviour upon alterations in GFAPisoform expression, a full characterization of changed behaviour has not yet been performed.

In this study, we investigated how manipulation of GFAPisoform expression affects human glioma cell invasion and growth dynamics ex vivo and in vivo. We longitudinally monitored the growth patterns of a total of twelve clones of U251-MG glioma cells depleted from either the GFAP $\alpha$ or the GFAP $\delta$ isoform in ex vivo organotypic mouse brain slices and in mouse brains in vivo with intravital imaging. We show that manipulation of the GFAPnetwork strongly affects the motility of glioma cells and tumour growth patterns. GFAP $\delta$-KO cells form denser tumours, have increased motility compared to control tumours and migrate randomly, whereas GFAPa-KO cells show a more diffuse growth pattern and migrate more persistently towards the brain parenchyma.

\section{Results}

GFAPisoform expression differs between low- and high-grade gliomas. Using differential gene expression analysis of RNA sequencing data from the The Cancer Genome Atlas (TCGA, https://www.cancer. gov/tcga), we previously showed that the ratio of splice variants GFAP $\alpha$ and GFAP $\delta$ differs between low gradeand high-grade gliomas ${ }^{6}$. Since this publication, 37 additional patient samples were included in the TCGA database. We therefore re-analysed the RNA sequencing data of the updated TCGA cohort and confirmed our previously reported findings. Whereas canonical splice variant GFAPa was significantly decreased in grade IV glioma compared to lower grades glioma (grade II and III) (Supplementary Fig. 1a), the expression of alternative splice variant GFAP $\delta$ was not different between the different grades (Supplementary Fig. 1b). Thus, there is an increased dominance of GFAP $\delta$ in high- versus lower-grade glioma, as illustrated by the significant increase in the GFAPS/a ratio (Fig. 1a).

Modification of GFAPisoform expression using CRISPR-Cas9. To understand how the different ratios of GFAP $\delta / \alpha$ affect the behaviour of the tumour cells, we modified GFAPisoform expression in the U251-MG human glioma cell line using CRISPR-Cas9 technology, as previously performed in Ref. ${ }^{7}$. A set of two single guide RNAs (sgRNAs) were used to delete the DNA region encoding the 41 or 42 amino acid tail of GFAP $\alpha$ and GFAP $\delta$, respectively. To create a GFAP $\alpha$ knockout (KO), the intronic regions before and after exon 8 and 9 were targeted, whereas the GFAP $\delta$-KO cells were created by flanking the intronic regions before and after exon 7a (Fig. 1b, Supplementary Fig. 2b,d). In addition to the six cell clones previously generated in $\operatorname{Ref}^{7}$ (CRISPR set A: CTL1, CTL2, GFAP $\delta$-KO1, GFAP $\delta$-KO2, GFAPa-KO1, GFAPa-KO2), we engineered six extra cell clones a using different set of sgRNAs (CRISPR set B: CTL3, CTL4, GFAP $\delta$-KO3, GFAP $\delta$-KO4, GFAPa-KO3, GFAPa-KO4) to create a total of twelve clones. Exonic depletion was confirmed with polymerase chain reaction (PCR) (Supplementary Fig. 2a,c) and sequencing (Supplementary Fig. 2b,d). Targeting the exonic region led to a significant decrease in mRNA levels of the corresponding isoform (Supplementary Fig. 2e,f,g) and an increase (GFAPa-KO) or decrease (GFAP $\delta$-KO) of the GFAP $\delta / \alpha$ mRNA ratio (Fig. 1c) and the GFAP $\delta /$ GFAPpan protein ratio (Fig. 1d,e, Supplementary Fig. 2h,i). The cell clones showed normal IFnetwork formation, except for GFAPa-KO clone 3, where occasional network collapses were observed (Supplementary Fig. 2j). This GFAPa-KO clone 3 had the highest GFAPS/GFAPpan protein ratio (Fig. 1d), confirming that there is a limit to the level of GFAP $\delta$ that can be incorporated into the network ${ }^{20,30}$.

Depletion of GFAPisoforms increases cell invasion in ex vivo organotypic brain slices. To study how modulation of the GFAPnetwork affects cell invasion in a physiologically relevant environment, we adapted the ex vivo organotypic brain slice model described by Ref. ${ }^{31}$. Ex vivo, $350 \mu \mathrm{m}$ thick brain slices of p1517 mouse pups were prepared and cultured in an air-liquid interface. The twelve cell clones were transduced with $\mathrm{H} 2 \mathrm{~B}-\mathrm{mNeonGreen}$ to visualise the nuclei and were injected into the lateral ventricles of the organotypic brain slice using a micromanipulator. The twelve H2B-mNeonGreen expressing cells (4 CTLs, 4 GFAP $\delta$-KOs, 4 GFAPa-KOs ) were co-injected with an internal control clone (I-CTL, CRISPR set A: CTL1, CRISPR set B: CTL3) that expressed H2B-mCherry. The brain slices injected with U251-MG cells were kept in culture for one week (Fig. 2a). Upon fixation of the ex vivo slices, we applied whole-mount immunofluorescent staining for laminin and used RapiClear tissue clearing ${ }^{32}$. Subsequently, we used confocal imaging to create a three-dimensional (3D)-reconstruction of the invasion patterns of the cells in the brain slice (Fig. 2b, Supplementary Fig. 3). Laminin expression was not only observed along the blood vessels but deposits produced by the glioma cells were also observed at the injection site where the cell density was the highest (Fig. 2b, Supplementary Fig. 3). We used this laminin expression pattern to distinguish cells within the tumour core from cells that had invaded into the mouse brain tissue (Supplementary Fig. 3).

First, we compared the distribution of nuclei of the CTLs, GFAP $\delta$-KOs, and GFAPa-KOs to that of the I-CTLs. We calculated the distance of every individual nucleus from the boundary of the tumour core and 


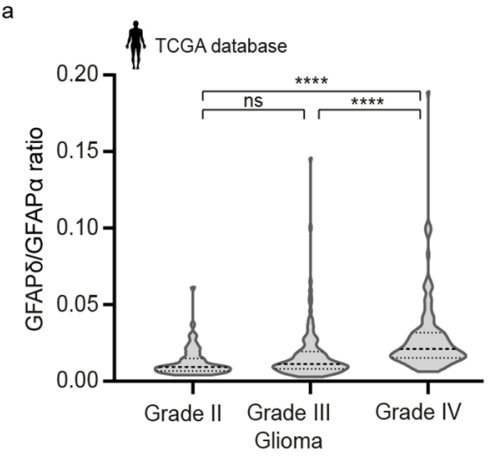

d

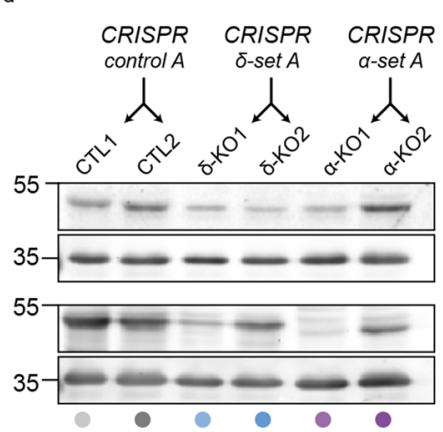

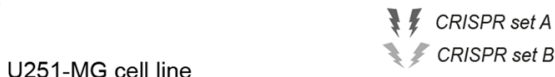

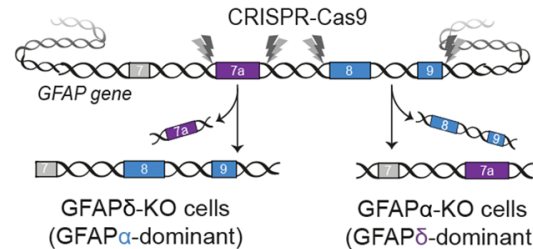

$c$

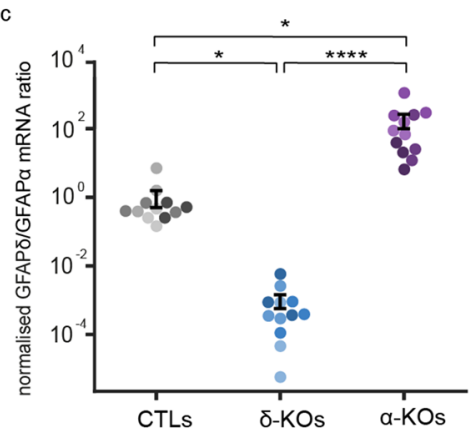

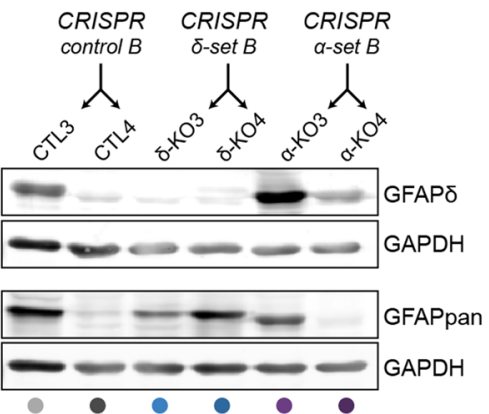

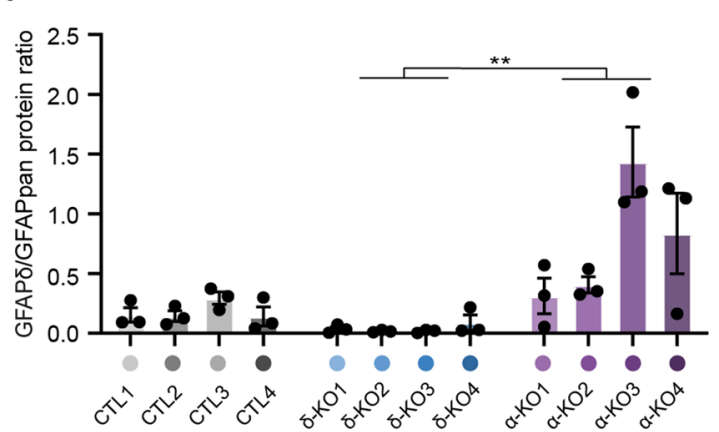

Figure 1. GFAPS/GFAP $\alpha$ ratio in the TCGA database and generation of GFAP isoform KO clones to regulate the GFAP $\delta / G F A P \alpha$ ratio in U251-MG glioma cells. (a) Violin plots of the GFAP $\delta / G F A P \alpha$ ratio in tumour samples of grade II $(n=64)$, grade III $(n=130)$, and grade IV $(n=153)$ astrocytoma, obtained from normalised isoform expression data of the TCGA database. Significance was determined using a Kruskal-Wallis test followed by a Dunn's multiple comparisons test. (b) Schematic illustration of the GFAP gene with the CRISPRCas9-targeted locations to generate GFAP $\delta$ - and GFAP $\alpha-K O$ cell clones. GFAP $\delta$-KO and GFAP $\alpha-K O$ cell clones were generated using two sets of sgRNAs (CRISPR set A and B) and four clones per isoform-KO were selected and characterised, leading to a total of 12 cell clones. (c) GFAPS/GFAPa mRNA ratio of the GFAP isoform KO cells and controls, represented on a $\log 10$ scale. Depletion of exon $7 \mathrm{a}(\mathrm{GFAP} \delta$-KO) leads to a decrease in the GFAPS/GFAPa ratio compared to the control cells, whereas depletion of exons 8 and 9 (GFAPa-KO) leads to an increase in the ratio. $\mathrm{n}=12$ biological repeats per group, derived from 4 clones per condition represented with different colour hues. Significance was determined using a Kruskal-Wallis test followed by a Dunn's multiple comparisons test. (d) Protein levels of GFAP $\delta$ and all GFAP isoforms (GFAPpan) in the 12 different cell clones generated with the different CRISPR sets (CRISPR control A, CRISPR $\delta$-set A, CRISPR $\alpha$-set A, CRISPR control B, CRISPR $\delta$-set B, CRISPR $\alpha$-set B) determined with Western blot. Full-lenth blots are presented in Supplementary Fig. 6 (e) Quantification of GFAP $\delta / G F A P p a n$ levels in the 12 different cell clones. Significance was determined using a Kruskal-Wallis test followed by a Dunn's multiple comparisons test. The data is shown as mean \pm S.E.M, ${ }^{*} \mathrm{p}<0.05,{ }^{* *} \mathrm{p}<0.01,{ }^{* * *} \mathrm{p}<0.001,{ }^{* * * *} \mathrm{p}<0.0001, \mathrm{~ns}=$ not significant.

plotted the distribution of the cells within different distance bins (Fig. 2c). As expected, the distribution plot of H2B-mNeonGreen expressing CTLs overlapped with that of the H2B-mCherry expressing I-CTLs (Fig. 2d, Supplementary Fig. 4a). The distribution of nuclei of the GFAP $\delta$-KO cells slightly deviated from the I-CTL line (Fig. 2e,f, Supplementary Fig. 4b), but the clearest alteration in distribution was observed in the GFAPa-KO cells. Whereas I-CTL cells have the highest density of cells in the tumour core, the GFAPa-KO cells showed a more diffuse growth pattern (Fig. 2g, Supplementary Fig. 4c). When plotting the distribution of cells, a shift in cell density towards the tumour border and tissue was observed (Fig. 2h), indicating more invasion. We next quantified the percentage of cells in the tissue as a measure for invasion and indeed observed a higher percentage of invading GFAPa-KO cells in comparison to its I-CTL and in comparison to the CTLs (Fig. 2i). Whereas GFAP $\delta$-KO had similar percentages of invading cells compared to its I-CTL, a higher percentage of invading cells was measured in comparison to the CTLs (Fig. 2i).

To confirm the effect of downregulating GFAPa on tumour distribution patterns, we repeated the ex vivo organotypic brain slice invasion experiment with U251-MG cells transduced with an shRNA against the 3'UTR of GFAPa (Supplementary Fig. 5a), as earlier published in Ref. ${ }^{5}$. Targeting GFAP $\alpha$ at the mRNA level led to a diffuse growth pattern and more invading cells, similar to the observations seen in CRISPR-Cas9 modified cells (Supplementary Fig. 5).

Depletion of GFAP $\alpha$ isoform leads to more diffuse tumours in vivo. Next, we aimed to study the GFAP-modulated cells in an in vivo setting where a functional vasculature is present, and where it is possible to follow tumour progression over time. We used intravital microscopy (IVM), which allows to longitudinally visualise tumour cell behaviour at the single-cell level in a living organism ${ }^{33}$. Per condition, we separately injected 
a

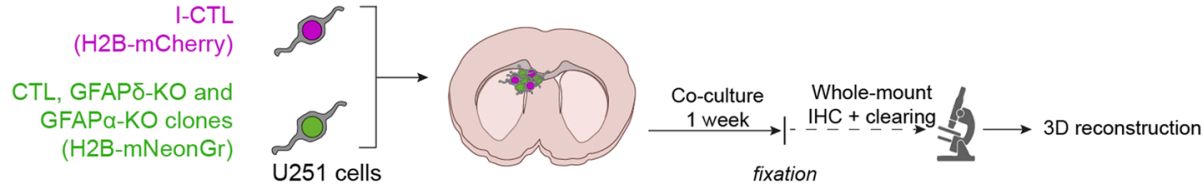

b
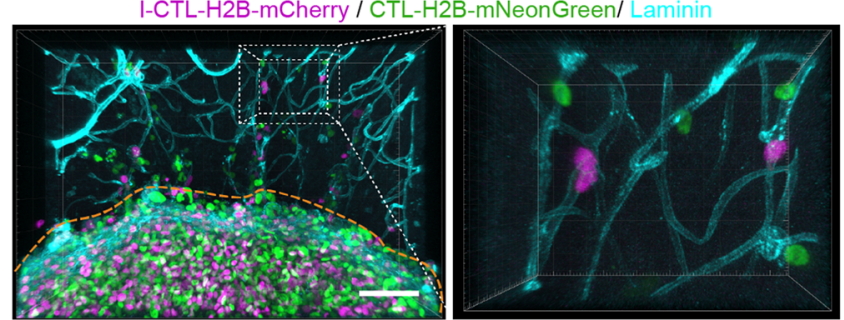

Scale bar $=100 \mu \mathrm{m}$
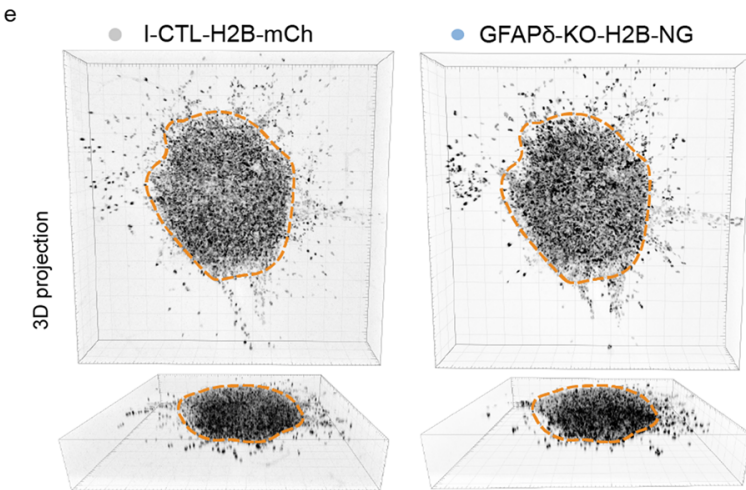

g

के

- I-CTL-H2B-mCh
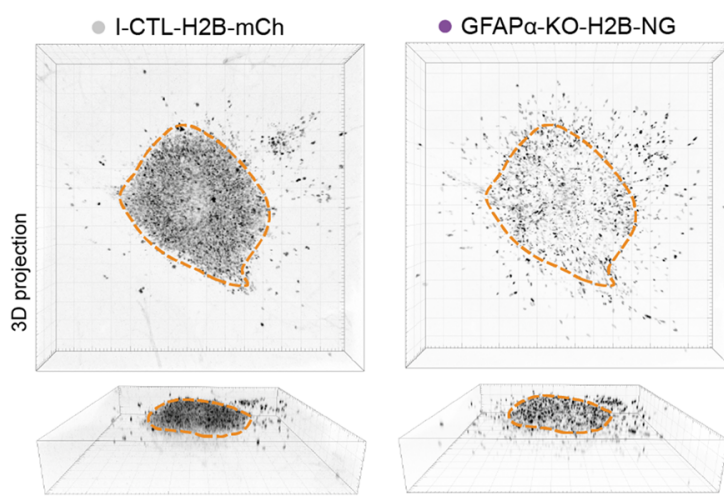

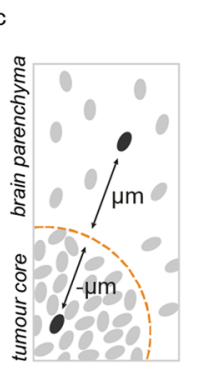

-CTL / GFAPס-KO/Lan
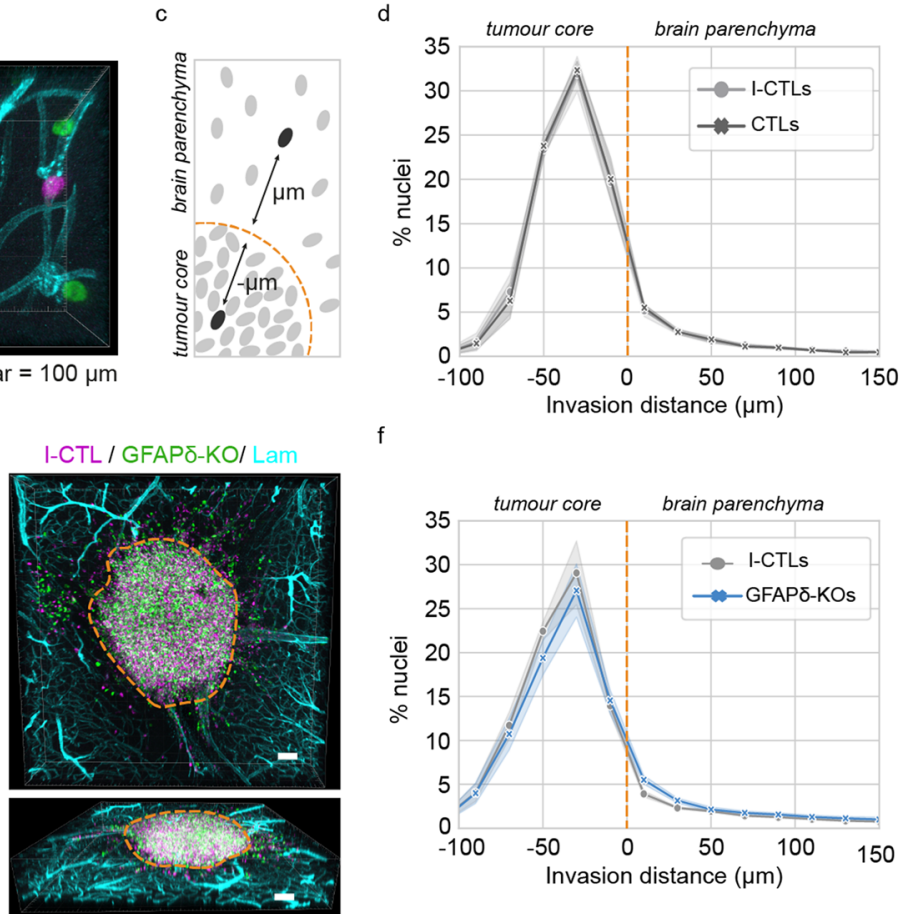

h

I-CTL / GFAPa-KO/ Lam

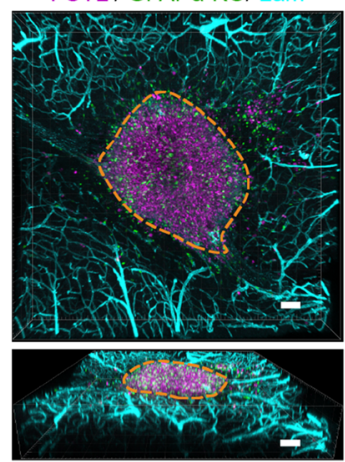

Scale bar $=100 \mu \mathrm{m}$

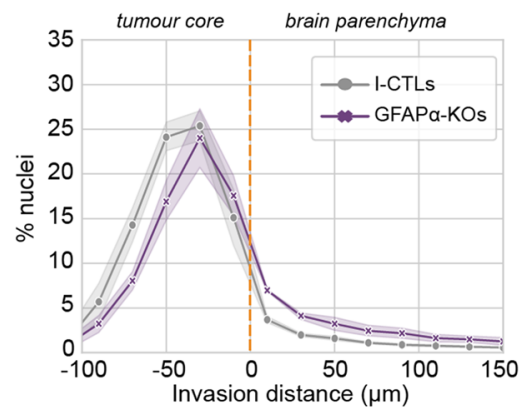

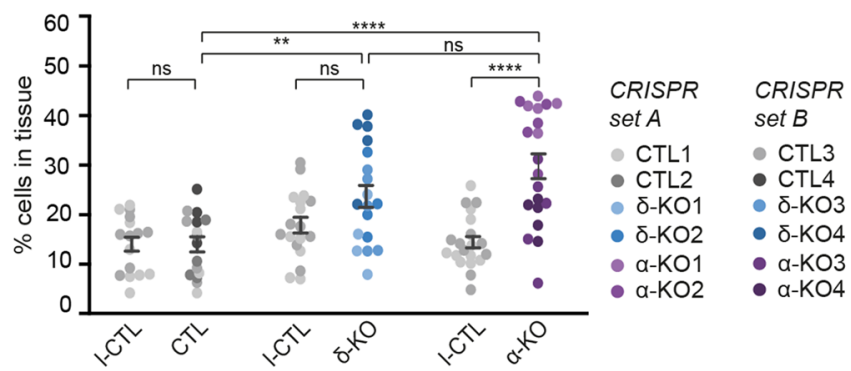


4Figure 2. Modification of GFAP isoform expression affects macroscopic growth patterns in organotypic brain slice cultures. (a) Schematic of experimental set-up: H2B-mNeonGreen expressing control (CTL), GFAP $\delta$-KO and GFAP $\alpha-\mathrm{KO}$ cell clones are injected in organotypic brain slices together with an H2B-mCherry expressing internal control (I-CTL) and co-cultured for one week. After fixation, whole-mount immunofluorescent staining, and clearing, confocal images are used to create a 3D reconstruction of the invasion patterns. (b) Representative image of I-CTL1 (magenta) and CTL1 (green) cells within the organotypic brain slice model. Invading cells are mainly found around the mouse brain vasculature (laminin, cyan). Laminin deposits in the tumour core can be used to distinguish stationary cells from cells invading the tissue, indicated with the orange dotted line. (c) Schematic depicting the method used to quantify the distribution of nuclei in the organotypic brain slices. (d) Distribution of nuclei of all I-CTL and CTL cells in the organotypic brain slices ( $n=16$ independent experiments, 4 different clones). (e) Representative images of invasion pattern of GFAP $\delta$-KO clone 1 and I-CTL 1. (f) Distribution of nuclei of all GFAP $\delta$-KO and I-CTL cells in the organotypic brain slices ( $\mathrm{n}=18$ independent experiments, 4 different clones). (g) Representative image of the invasion pattern of GFAPa-KO clone 2 and I-CTL 1. (h) Distribution of nuclei of all GFAPa-KO cells and I-CTL cells in the organotypic brain slices ( $\mathrm{n}=20$ independent experiments, 4 different clones). (i) Quantification of the percentage of invaded cells per condition, $n=16$ (CTLs), $n=18$ (GFAP $\delta$-KO), and $n=20$ (GFAP $-\mathrm{KO}$ ) injected organotypic brain slices derived from 4 different clones (CRISPR set A and B) per condition. Significance was determined using a two-way ANOVA followed by Tukey's multiple comparisons test. Scale bar $=100 \mu \mathrm{m}$. The data is shown as mean \pm S.E.M, ${ }^{\star} \mathrm{p}<0.05,{ }^{* *} \mathrm{p}<0.01,{ }^{* * *} \mathrm{p}<0.001,{ }^{\star * * *} \mathrm{p}<0.0001$, ns $=$ not significant. $\mathrm{NG}=$ $\mathrm{mNeonGreen,} \mathrm{mCh}=\mathrm{mCherry}$, Lam $=$ laminin.

two H2B-mNeonGreen expressing clones with the most extreme GFAP $\delta / \alpha$ ratio (CTL 1, GFAP $\delta$-KO 2, and GFAPa-KO 2 from CRISPR set A, CTL 3, GFAPS-KO 3, and GFAPa-KO 4 from CRISPR set B) into NODScid IL2Rgnull (NSG) mice. Tumour development was followed using a cranial imaging window (CIW). An overview image of the tumour at the endpoint was taken, when a well-established tumour with similar size had formed (Fig. 3a,b). To quantify the tumour density, we calculated the number of individual cells in the total tumour area. We observed that tumours generated by the GFAP $\delta$-KO were significantly denser than tumours generated by the GFAP $\alpha-\mathrm{KO}$ cells (Fig. 3b,c). This suggested that GFAP $\alpha-\mathrm{KO}$ cells have a more diffuse growth pattern compared to GFAP $\delta$-KO cells.

Depletion of GFAP isoforms increases motility and alters invasion patterns in vivo. To further gain insight into the migratory behaviour of GFAP-modulated glioma cells in vivo, we again made use of the CIW to longitudinally study invasive behaviours at the single-cell level. At endpoint, a series of time-lapse $\mathrm{z}$-stack images of the tumour was acquired for $6 \mathrm{~h}$ with a time interval of $45 \mathrm{~min}$ (Fig. $4 \mathrm{a}$ ). The movement of individual glioma cells was determined by tracking their migration path over time in $3 \mathrm{D}$-reconstructed time-lapse movies (Fig. 4b). Data concerning migration velocity, speed, persistence, and directionality were extracted from the tracks. This showed that depletion of either GFAP $\delta$ or GFAPa isoform leads to an increase in the percentage of motile cells compared to the CTL (Fig. 4c). While the GFAP $\delta$-KO cells migrate faster than the CTL cells (Fig. 4d,e), they move with less persistence compared to the GFAPa-KO and CTL cells (Fig. 4f). Considering that directionality is an important factor for invasion, we analysed the directionality patterns in each tumour type and determined whether the cells were migrating towards the tumour core or the brain parenchyma ${ }^{34}$. This demonstrated that GFAPa-KO cells migrate more towards the brain parenchyma while the CTL cells and GFAP $\delta$-KOs migrate more randomly (Fig. 4b,g). Indeed, this data is in line with our observation that GFAPa-KO tumours are more diffuse than GFAP $\delta$-KO tumours (Fig. 3b,c).

It has been recently shown that nucleus stiffness and cell deformability plays an important role in cell motility. For instance, to move in a three-dimensional ECM, the nucleus of a cell must squeeze through the narrow spacing within the brain parenchyma ${ }^{35-37}$. In our model, we observed a significant increase in the nuclear axis length of GFAPa-KOs compared to CTLs (Fig. 4h), which may contribute to the increased ability of GFAPa-KO to infiltrate the brain parenchyma.

\section{Discussion}

The invasive nature of glioma makes the disease highly aggressive and hard to treat. Therefore, precisely understanding the mechanisms driving invasion of glioma cells is crucial for the development of new anti-invasive treatment strategies. In this study, we investigated the role of GFAPisoforms in glioma cell invasion, using an organotypic brain slice invasion model and intravital imaging through a CIW. We show that the GFAP $\delta / \alpha$ ratio affects the macroscopic growth patterns of glioma cells both ex vivo and in vivo by regulating cell migration speed, directionality, and persistence. Importantly, we demonstrate that GFAP $\delta$-KO cells show increased motility compared to CTL and GFAPa-KO cells in vivo, but move randomly. GFAPa-KO cells, on the other hand, move more persistently and have a strong tendency to migrate towards the brain parenchyma. These dynamics of the GFAPa-KO cells lead to a more diffuse infiltration pattern into the brain parenchyma.

Earlier studies that investigated the role of GFAP in cell motility and migration have been somewhat inconsistent. As such, GFAP expression has been linked to both higher ${ }^{38,39}$ and lower ${ }^{5,40,41}$ velocities of cell migration. Also, shRNA mediated knockdown of GFAPa decreased cell velocity in an earlier in vitro study ${ }^{5}$, which is inconsistent with the phenotype we describe here. The effect of GFAP depletion on cell behaviour might not only be isoform dependent, but also influenced by the cell-environmental context ${ }^{34}$, which may explain discrepancies between earlier studies performed in 2D. Our study is the first, to our knowledge, to investigate the role of GFAP and its isoforms within the physiological context of the brain. We find that depletion of both isoforms leads to an 
a

Tumour cell injection (H2B-mNeonGreen) and CIW implantation
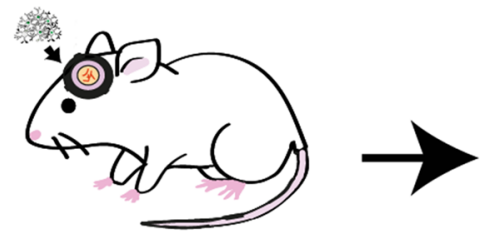

b
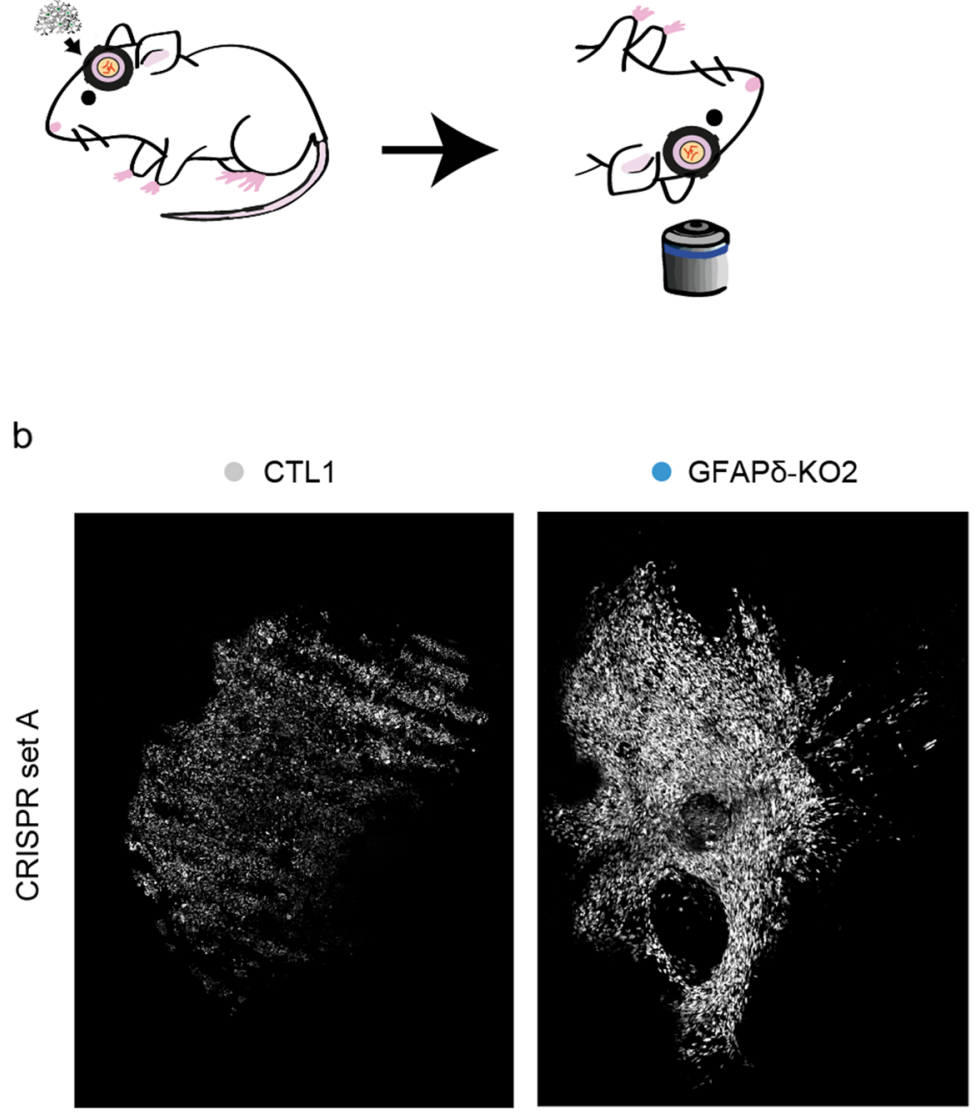

Intravital

imaging at endpoint

CTL3
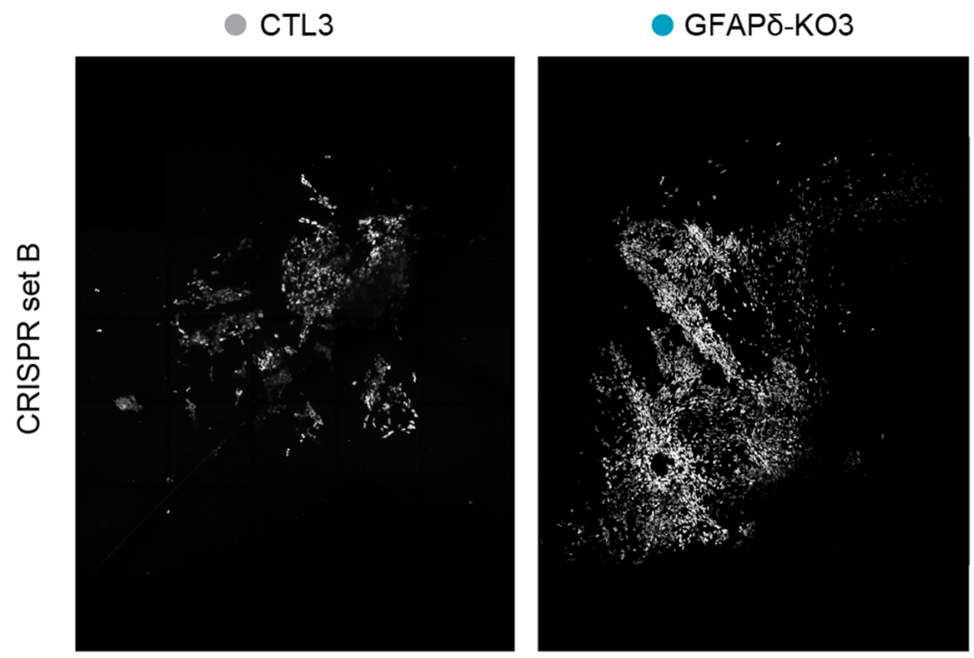

C

Tumour density
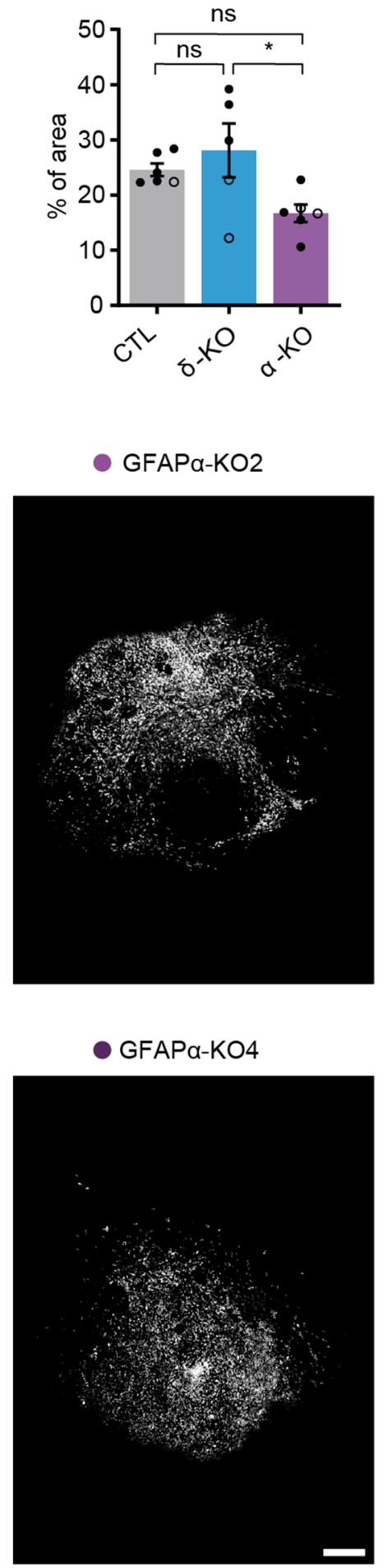

Scale bar $=500 \mu \mathrm{m}$

Figure 3. In vivo tumour growth dynamics in GFAP-modulated tumours. (a) Schematic overview of the experimental setup. U251-MG GFAP-modulated cell clones expressing H2B-mNeonGreen were implanted in the brain of NSG mice under a CIW. Timelapse intravital imaging was performed through a CIW to study the tumour growth dynamics of each tumour type. (b) Representative 3D reconstructed tile-scans showing distinct tumours generated by different GFAP-modulated clones. Two clones engineered with different CRISPR-Cas9 sgRNAs are presented (CTL1, GFAP $\delta$-KO2 and GFAP $\alpha-K O 2$ from CRISPR set A and CTL3, GFAP $\delta$-KO3 and GFAP $\alpha-K O 4$ from CRISPR set B). Scale bar $=500 \mu \mathrm{m}$ (c) Quantification of tumour density for each indicated tumour type. $\mathrm{n}=6$ (CTLs), $\mathrm{n}=5$ (GFAP $\delta$-KO), and n=6 (GFAP $\alpha-\mathrm{KO})$ mice. All tumours were imaged when they had filled half the imaging window (endpoint), which was between 13 and 35 days after the cranial window implantion, except for one CTL1 tumour which reached endpoint after 72 days. Black dots represent clones from CRISPR set A (CTL1, GFAP $\delta$-KO2, GFAP $\alpha$-KO2) and white dots represent clones from CRISPR set B (CTL3, GFAP $\delta$-KO3, GFAP $\alpha-K O 4)$.The data is shown as mean \pm S.E.M, ${ }^{*} \mathrm{p}<0.05,{ }^{* *} \mathrm{p}<0.01,{ }^{* * *} \mathrm{p}<0.001$, ${ }^{* * * *} \mathrm{p}<0.0001, \mathrm{~ns}=$ not significant, one-way ANOVA followed by Tukey's multiple comparisons test. 
a

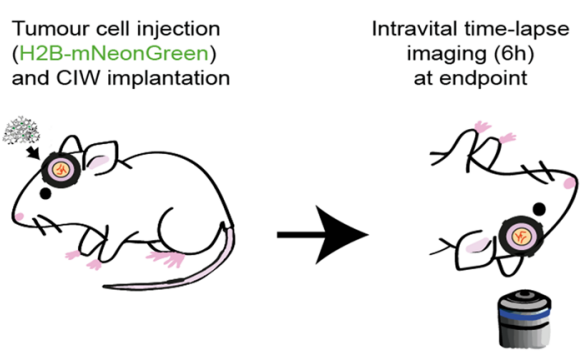

b

- CTL1
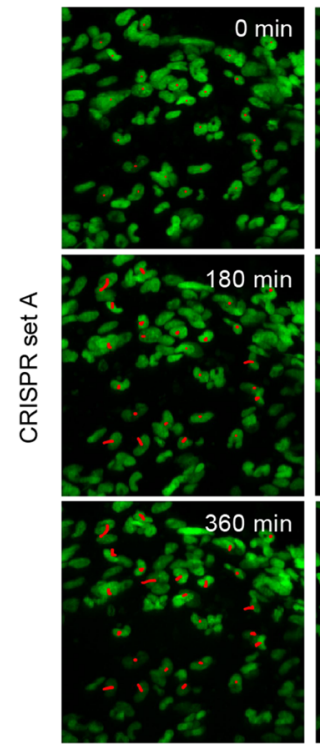

Brain

parenchyma

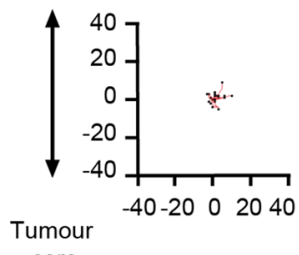

- GFAPס-KO2
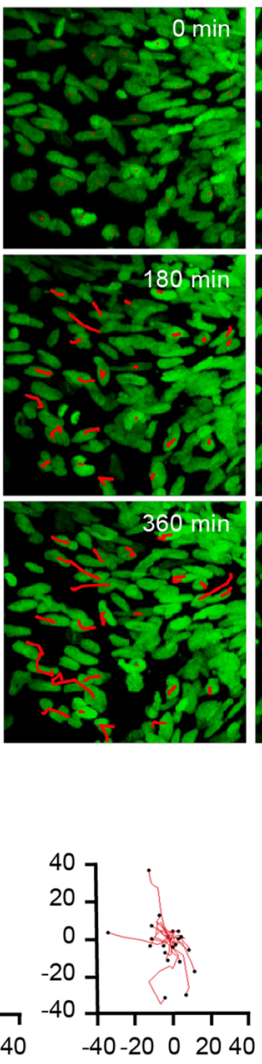

$-40-2002040$
- GFAPa-KO2
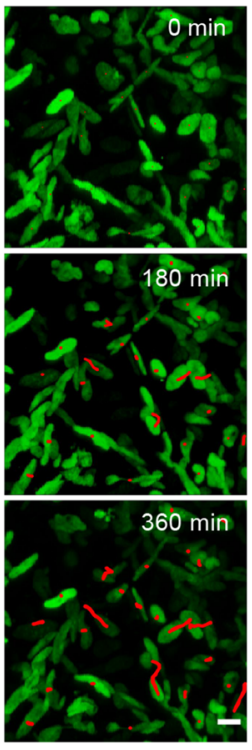

Scale bar $=20 \mu \mathrm{m}$

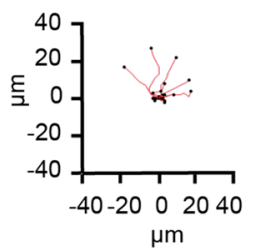

c
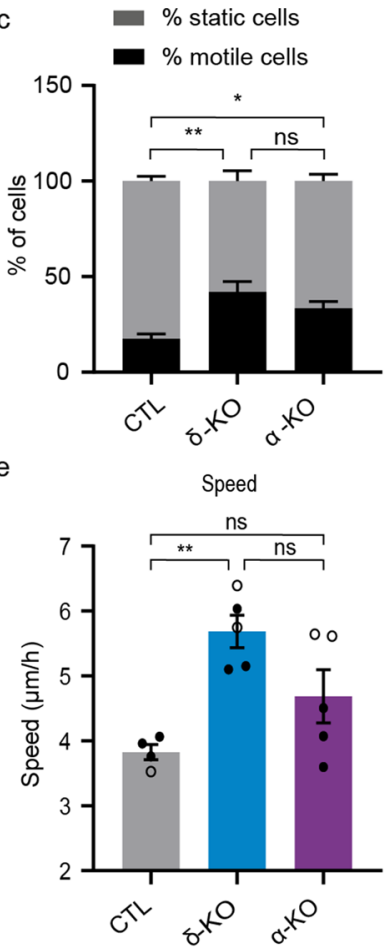

g $\begin{gathered}\text { Centre of mass } \\ \text { displacement }\end{gathered}$

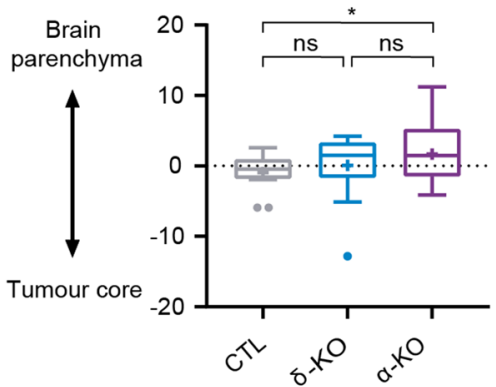

d
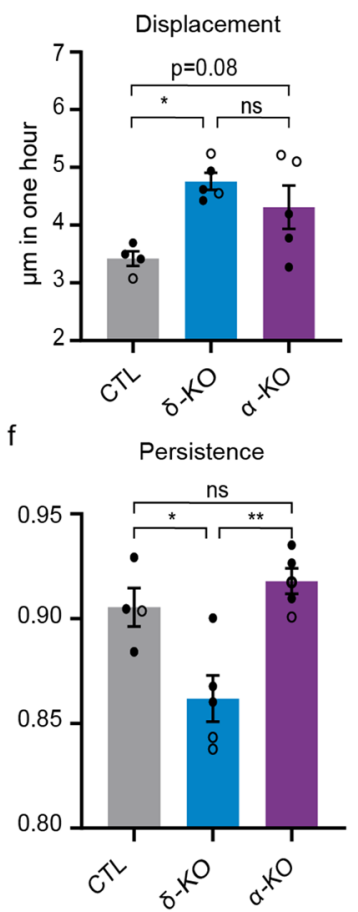

h

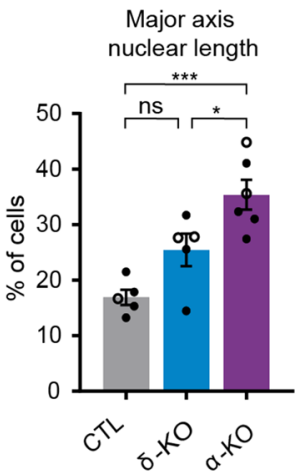

Figure 4. In vivo migratory behaviour of tumour cells with different GFAP $/ \alpha$ ratios. (a) Schematic representation of implantation of CIW and intravital time-lapse imaging over $6 \mathrm{~h}$ with an interval of $45 \mathrm{~min}$. (b) Representative still images from a time-lapse movie showing migratory tumour cells in different GFAP -modulated tumours (CTL1, GFAP $\delta$-KO2, GFAPa-KO2). Red lines highlight individual tumour cell tracks. Scale bar $=20 \mu \mathrm{m}$. Corresponding plots represent tracks from a common origin showing the direction of the tumour cells either towards the tumour core or the brain parenchyma. (c) Percentage of motile (cell displacement $>2 \mu \mathrm{m} /$ hour) and static cells for each tumour type. (d) Quantification of cell displacement of motile cells for the indicated tumour type. (e) Cell speed of motile cells for the different cell clones $(\mu \mathrm{m} / \mathrm{h})$. (f) Cell persistence of motile cells in the different cell clones. Black dots represent clones from CRISPR set A (CTL1, GFAP $\delta$-KO2, GFAP $\alpha-K O 2)$ and white dots clones from CRISPR set B (CTL3, GFAP $\delta$-KO3, GFAPa-KO4). (g) Tukey-style whiskers plot of the centre of mass displacement of individual positions of each condition. (h) Quantification of nuclear cell length in the different cell clones. \% of cells with a length higher than $30 \mu \mathrm{m}$ is represented. $n=4$ (CTLs), $n=5$ (GFAP $\delta$-KO), and $n=5$ (GFAPa-KO) CIW mice implanted with 2 different cell clones, one of each CRISPR set. The data is shown as mean \pm S.E.M, ${ }^{*} \mathrm{p}<0.05,{ }^{* *} \mathrm{p}<0.01,{ }^{* * *} \mathrm{p}<0.001$, ${ }_{* * * *} \mathrm{p}<0.0001, \mathrm{~ns}=$ not significant, one-way ANOVA or two-way ANOVA followed by Tukey's multiple comparisons test. 
increase in the number of motile cells, as well as an increase in cell displacement and speed (Fig. 4c-e). Therefore, cell-intrinsic motility and velocity may not be dependent on the GFAP $\delta / \alpha$ ratio, but on modification of the GFAPnetwork in general. In contrast to motility and velocity, we demonstrate that directionality and persistence of cell migration is GFAPisoform dependent. Previous studies have shown that the IFnetwork can promote migration persistence by modulating microtubule organization and cell polarity ${ }^{38,42-44}$. Whether the absence of GFAPa or dominance of GFAP $\delta$ regulates directional migration similarly remains to be elucidated. The directionality of migration and invasion can also be steered by extrinsic factors ${ }^{45}$ such as ECM composition and topology ${ }^{45}$. For instance, adhesive interaction of the cell with the extracellular microenvironment as well as remodelling of the ECM are required to migrate efficiently through the extracellular space ${ }^{46}$. MMPs are responsible for the degradation of a large range of ECM proteins and GBM cells have been shown to overexpress MMP2 and $9^{47}$. In line with this, we showed in our previous work that modulation of the GFAPa isoform affects genes involved in the compositions of the ECM and extracellular space ${ }^{6}$. In addition, we previously demonstrated that GFAPa-KO cells produce more laminin and overexpress MMP2 by activating signaling pathways up- and downstream of dual-specificity phosphatase 4 (DUSP4) ${ }^{5,7}$. This might contribute to the higher ability of these cells to invade the brain parenchyma persistently. Additionally, it has been shown that immune cells, including macrophages and microglia, also promote glioma invasion ${ }^{48-50}$. Considering that our experiments were performed in immunodeficient NOD-SCID mice, it remains to be elucidated whether interactions with immune cells potentially affect the invasive behaviour of glioma cells with different GFAP $\delta / \alpha$ ratios.

The findings of this study contribute to our understanding on how a switch in the GFAP $\delta / a$ ratio in grade IV glioma patients may affect the aggressiveness of these tumours. Increased dominance of GFAP $\delta$ in grade IV glioma tumours has been reported by multiple studies ${ }^{6,25,26,28}$ (reviewed in Ref. ${ }^{29}$ ), and was confirmed by analysis of the updated TCGA database (Fig. 1a). Similarly, Brehar and colleagues reported that patients with highly invasive tumours, based on pre-operative MRI, had increased percentages of GFAP $\delta$ positive cells ${ }^{27}$. Glioma tumours are known to be highly heterogeneous. This heterogeneity appears to be not only between patients but also between single cells within a tumour ${ }^{51-53}$, therefore it is likely that the same tumour is composed of a mix of cells with a high and low GFAP $\delta / \alpha$ ratio and these distinct cell populations may contribute to different behaviour. Together, it can be hypothesised that a larger population of high GFAP $\delta / a$ ratio cells in grade IV tumours contributes to infiltration of the brain parenchyma and subsequent relapse after therapy. Further work is needed to understand the contribution of the GFAP $\delta / \alpha$ ratio to the infiltrative growth of low and high-grade glioma tumours in clinical samples, for instance using intravital imaging in patient-derived xenografts ${ }^{54}$.

How the shift in GFAPisoform expression is established in grade IV tumours is currently unknown. Alternative splice events are known to occur more frequently in tumour tissue in comparison to non-malignant cells $^{55}$, and dysregulation of the splicing machinery drives glioma aggressiveness ${ }^{56}$. Recently, it was discovered that hypoxia can induce adult-to-foetal splicing transitions in glioma, regulated by muscle blind-like proteins $(\mathrm{MBNL})^{57}$. Hypoxia is considered an important driver of glioma invasion and is typically associated with grade IV gliomas ${ }^{58,59}$. GFAP has multiple predicted binding motifs for the hypoxic-associated splicing factor MBNL ${ }^{60}$, The link between hypoxia, GFAP alternative splicing, and cell invasion remains to be investigated.

In summary, our work demonstrates the importance of GFAPisoforms in fine-tuning glioma invasion and tumour dynamics. Together, the increased understanding of the mechanisms driving the invasive behaviours of different GFAP positive populations that form glioma tumours will help develop better anti-invasive therapeutic strategies in the future.

\section{Materials and methods}

Cell lines and culture. The cell identity of malignant glioma cell line U251-MG (obtained from Lars Ruether, Institut für Neuropathologie, Universitätsklinikum Münster, Münster, Germany) was confirmed by short terminal repeat analysis (Eurofins Scientifc, Luxembourg city, Luxembourg). All cells were cultured in DMEM high glucose (Gibco 41966052) mixed 1:1 with Ham's F10 nutrient mix (Gibco 22390025) supplemented with 10\% fetal bovine serum (Gibco 10270106/Biowest $\mathrm{S} 181 \mathrm{H}$ ) and $1 \%$ penicillin/streptomycin (Gibco 15140122 ) at $37^{\circ} \mathrm{C}$ in a humidified incubator with $5 \% \mathrm{CO}_{2}$. Cells were routinely tested negative for mycoplasma contamination.

Mice.

For the generation of organotypic slice cultures, 15-17 day-old C57BL6J male and female mice were used. C57BL6J mice were obtained from Charles Rivers Laboratories and bred in-house. The animals were kept under a normal 12:12 h light-dark cycle with lights off at 19:00, at room temperature $\left(21 \pm 2{ }^{\circ} \mathrm{C}\right)$ and at $40-70 \%$ humidity conditions, and were fed with chow and water ad libitum.

For intravital imaging experiments, NOD-Scid IL2Rgnull male and female mice (NSG), aged 8 to 20 weeks at the time of cranial window implantation were used. Mice were housed in individually ventilated cage and received food and water ad libitum.

All experimental protocols used in this manuscript were in accordance with ARRIVE guidelines, national regulations, and ethical guidelines and were approved by the Centrale Commissie Dierproeven (CCD) and the Instantie voor Dierenwelzijn (IvD).

TCGA RNA sequencing data collection and analysis. Expression data of GFAP splice variants from the Cancer Genome Atlas (TCGA) was extracted using the TSVdb webtool (http://tsvdb.com) ${ }^{61}$. Normalised RSEM (RNA-Seq by Expectation aximization) count estimates from TCGA Lower Grade Glioma (TCGA-LGG) and glioblastoma multiforme (TCGA-GBMs) projects were extracted and matched with the sample ID to clinical data on histological subtype and malignancy grade downloaded from the TCGA database: https://www. cancer.gov/tcga. The GFAP $\alpha$ and GFAP $\delta$ normalised expression levels and GFAP $\delta / \alpha$ ratios were compared in data from 64 grade II astrocytomas, 130 grade III astrocytomas and 153 GBMs. 
Generation of CRISPR-Cas9 plasmids. Single guide RNAs (sgRNAs) targeting the intronic regions before and after exon7a (GFAP $\delta$-KO) or exon 8 and 9 (GFAPa-KO) were designed using web resources of the Broad Institute (http://tools.genome-engineering.org//) ${ }^{62}$ or CRISPOR.org (http://crispor.tefor.net/) ${ }^{63}$ and were selected based on proximity to exons and MIT and CFD specificity score ${ }^{64}$. The sgRNA complementary oligonucleotide templates (Supplementary Table 1) were cloned into pSpCas9(BB)-2A-Puro (Addgene, \#48139) or pSpCas9(BB)-2A-GFP (Addgene, \#48138) plasmids after BbsI (Thermo Fisher Scientific) digestion. Plasmids were isolated using a Maxiprep kit (LabNed) and the sequence was verified by Sanger sequencing (Macrogen, Amsterdam, The Netherlands). Per GFAPisoform, two sets of CRISPR-Cas9 plasmids were generated (CRISPR $\delta$-set A, CRISPR $\delta$-set B, CRISPR $\alpha$-set A, CRISPR $\alpha$-set B, Supplementary Table 1). Empty plasmids were used as a control (CRISPR control A and CRISPR control B). The sgRNAs cloned into the pSpCas9(BB)-2A-Puro plasmid (CRISPR control A, $\delta$-set A, $\alpha$-set A) and the cell clones generated with these plasmids (CTL1, CTL2, $\delta$-KO1, $\delta$-KO2, $\alpha-\mathrm{KO} 1, \alpha-\mathrm{KO} 2)$ have been described in Ref. ${ }^{7}$. The sgRNA pairs cloned into the pSpCas $9(\mathrm{BB})-$ 2A-GFP plasmid (CRISPR control B, $\delta$-set B, $\alpha$-set B) and the cell clones generated with these plasmids (CTL3, $\mathrm{CTL} 4, \delta$-KO3, $\delta$-KO4, $\alpha-\mathrm{KO} 3, \alpha-\mathrm{KO} 4)$ are first described in this paper.

For cell transfection of the CRISPR-Cas 9 construct and clonal expansion, U251-MG cells were seeded at a density of 0.8 to $1.2 \times 10^{5}$ cells in an uncoated 6-well plate. Twenty-four hours after seeding, the sets of CRISPRCas9 plasmids ( $1 \mu \mathrm{g}$ DNA total) with the sgRNAs upstream and downstream of the targeted exons of the GFAP isoforms were co-transfected using polyethylenimine (PEI, $166 \mathrm{ng} / \mathrm{mL}$ final concentration). Cells transfected with the pSpCas9(BB)-2A-Puro plasmids (CRISPR set A) were treated with $1 \mu \mathrm{g} / \mathrm{mL}$ puromycin (Sigma-Aldrich, 58-58-2) $24 \mathrm{~h}$ after transfection and were selected for $96 \mathrm{~h}$. The drug-resistant pool was expanded and cell clones were generated by single-cell sorting cells into 96-well plates using fluorescence-activated cell sorting (FACS; FACSAria II Cell Sorter). Cells transfected with the pSpCas9(BB)-2A-GFP plasmids (CRISPR set B) were selected for GFP using FACS (FACSAria II Cell Sorter) $48 \mathrm{~h}$ after transfection. The GFP-positive pool was expanded and cell clones were generated by plating cells at low densities in 96 -well plates $(0.5$ cell/well). The 96 -well plates were inspected for colony formation and cell clones were expanded.

Selection of CRISPR-Cas9 targeted cell clones. PCR screening was used to identify cell clones in which the targeted DNA region in the GFAP gene was depleted. Genomic DNA was isolated from cell pellets of the cell clones. Cells were lysed in $5 \mathrm{mM}$ Tris $\mathrm{HCl}(\mathrm{pH} 8.8)$ at $95^{\circ} \mathrm{C}$ for $10 \mathrm{~min}$ and treated with proteinase $\mathrm{K}$ at $56^{\circ} \mathrm{C}$ for $30 \mathrm{~min}$. The CRISPR-Cas 9 targeted DNA region was amplified using primers described in Supplementary Table 1, using the FirePol PCR Master Mix (Solis BioDyne, 04-12-00S15). PCR products were separated on a 1.5\% agarose gel containing SYBR Safe (Thermo Fisher Scientific, S33102) and GFAPisoform KO clones were identified based on the presence of predicted smaller PCR products. Depletion of the targeted DNA region was confirmed by isolating the amplified DNA of the PCR product using the PureLink Quick Gel Extraction Kit (Thermo Fisher Scientific, K210012) and Sanger sequencing (Macrogen, Amsterdam, The Netherlands).

shRNA construct design. Lentiviral shRNA expression plasmids targeting GFAPa or non-targeting controls were generated as described in Ref. ${ }^{5}$. In short, lentiviral shRNA expression plasmids from the RNAi Consortium (TRC) Mission library were obtained from Sigma-Aldrich (TRCN0000083733) ${ }^{65}$. A human shRNA construct against nucleotides 2674-2694 in the $3^{\prime}$ untranslated region of the GFAPa transcript or a SHC002 non-targeting construct (NTC) with no homology to human sequences were cloned into the pLKO.1 backbone. It was attempted to create shRNAs targeting the transcript of GFAP $\delta$, however we were unsuccessful in significantly downregulating this isoform (data not shown).

Lentiviral production and transduction of cells. Lentiviruses encoding NTC or GFAP $\alpha$ shRNA were produced as described in Ref. ${ }^{5}$. U251-MG cells were transduced with lentiviral particles encoding NTC or GFAPa shRNA with a multiplicity of infection (MOI) of 0.5 . Three days after transduction, cells were selected by treatment with $1 \mu \mathrm{g} / \mathrm{mL}$ puromycin (Gibco, A1113803) to create stable cell lines.

All U251-MG GFAP-modulated cells (with CRISPR-Cas9 or shRNAs) and controls were transduced with lentiviruses to induce expression of H2B-mNeonGreen or H2B-mCherry. The pLV-H2B-mNeonGreen-IRES-puro plasmid was a gift from Dr. Hugo Snippert ${ }^{66}$, the pLenti6-H2B-mCherry plasmid was a gift from Torsten Wittmann (Addgene plasmid \# 89766). Lentiviral particles were produced with standard third-generation lentiviral protocol. In short, $2 \times 10^{7} 293$ T cells (ATCC, ATCC-CRL-11268) were plated in a $15 \mathrm{~cm}^{2}$ dish and transfected the next day with a total of $51.6 \mu \mathrm{g}$ DNA of an envelope plasmid (pMD2.G), packaging plasmids (pMDLg/pRRE and pRSV-Rev) and pLV-H2B-mNeonGreen-IRES-puro or pLenti6-H2B-mCherry plasmid using PEI (166 ng/ $\mathrm{mL}$ final concentration). The medium was replaced $24 \mathrm{~h}$ after transfection. After $48 \mathrm{~h}$, the medium containing virus particles were collected and filtered through a $0.22 \mu \mathrm{m}$ filter. The supernatants were ultracentrifuged at $22,000 \mathrm{rpm}$ (rotor $70 \mathrm{Ti}$, Beckman ultracentrifuge) at $16^{\circ} \mathrm{C}$ for $2 \mathrm{~h}$ and $40 \mathrm{~min}$. The pellet was resuspended in PBS $+0.5 \%$ BSA (Sigma), aliquoted and stored at $-80^{\circ} \mathrm{C}$ until further use. The viral titre was determined by transducing $293 \mathrm{~T}$ cells with a dilution series of the virus. The viral titre was estimated in transducing units (TU)/ $\mathrm{mL}$ by counting the number of transduced fluorescent cells $48 \mathrm{~h}$ after transduction. The GFAP-modulated cells were transduced with $\mathrm{H} 2 \mathrm{~B}-\mathrm{mNe}$ - $G$ reen and $\mathrm{H} 2 \mathrm{~B}-\mathrm{mCherry}$ lentiviral particles with an MOI of 1 . Cells were passaged once and positive cells were selected by keeping the cells in medium containing $1.5 \mu \mathrm{g} / \mathrm{mL}$ puromycin (H2B-mNeonGreen clones) or $10 \mu \mathrm{g} / \mathrm{mL}$ blasticidin (H2B-mCherry clones) for 3 days.

Western blot analysis. Total protein was extracted from cultured cells scraped in suspension buffer [0.1 M $\mathrm{NaCl}, 0.01 \mathrm{M}$ Tris $\mathrm{HCl}$ (pH 7.6), 0.001 M EDTA, and Complete EDTA-free protease inhibitor cocktail (Roche)] and sonicated $(2 \times 10 \mathrm{~s})$ in an ultrasonic bath. An equal amount of $2 \times$ SDS loading buffer [100 $\mu \mathrm{M}$ Tris ( $\mathrm{pH} 6.8)$, 
$4 \%$ SDS, 20\% glycerol, 5\% 2-ME, and bromophenol blue] was added to the cell suspension, samples were heated at $95^{\circ} \mathrm{C}$ for $5 \mathrm{~min}$ and DNA was broken down by pushing the sample through a 25 -gauge needle. Equal amounts of sample were loaded on a $10 \%$ SDS-page gel and proteins were separated by electrophoresis. Proteins were then blotted on a $0.45-\mu \mathrm{m}$ pore size nitrocellulose membrane (GE Healthcare) using a wet/tank transfer blotting system (Biorad, 170390). Membranes were blocked in blocking buffer ( $50 \mathrm{mM}$ Tris pH 7.4, $150 \mathrm{mM} \mathrm{NaCl}, 0.25 \%$ (w/v) gelatin, $0.5 \%$ Triton-X100) for $10 \mathrm{~min}$ and incubated with primary antibodies (Supplementary Table 2) in blocking buffer overnight at $4{ }^{\circ} \mathrm{C}$. Membranes were washed with TBS with $1 \%$ Tween (TBS-T) three times for $10 \mathrm{~min}$ and then incubated with secondary antibodies (Supplementary Table 2) in blocking buffer at room temperature for $1 \mathrm{~h}$. After three washing steps with TBS-T and one washing step with MilliQ, the membrane blots were scanned with the Odyssey Clx Western Blot Detection System (Li-Cor Biosciences). The backgroundcorrected signal intensity of bands corresponding to the GFAPpan and GFAP $\delta$ proteins were measured and normalised against the intensity levels of glyceraldehyde 3-phosphatedehydrogenase (GAPDH) bands on the same blots.

RNA isolation, cDNA isolation and real-time quantitative PCR. For RNA extraction of cultured cells, cells were seeded on poly-D lysine (PDL)-coated wells of a 24 -well plate at a density of $4 \times 10^{4}$ cells. After three days in culture, cells were lysed in TRIzol (Thermo Fisher Scientific, 15596026) and RNA was extracted using standard TRIzol-chloroform extraction methods. RNA concentration and purity were measured using Varioscan Flash (Thermo Fisher Scientific). 200 to 500 ng of RNA were used to prepare cDNA using the QuantiTect Reverse Transcription Kit (Qiagen, 205311) according to the manufacturer's protocol. The generated cDNA was used for real-time quantitative PCR using the SYBR Green Master mix in a QuantStudio 6 Flex Real-Time PCR system (Thermo Fisher Scientific, 4309155) using the primers listed in Supplementary Table 1. Expression values were calculated by transforming $\mathrm{Ct}$ values $\left(2^{-\mathrm{Ct}}\right)$ and were normalised to the mean value of the transformed $\mathrm{Ct}$ values of the reference genes GAPDH and Alu element Jurka (Alu-J).

Immunocytochemistry. For immunocytochemistry on cultured cells, cells were seeded on PDL-coated coverslips in a 24 -well plate at a density of $2 \times 10^{4}$ cells. After three days in culture, the cells were fixed in $4 \%$ paraformaldehyde (PFA) dissolved in phosphate buffer saline (PBS), pH 7.4 for $30 \mathrm{~min}$. Cells were washed in PBS, incubated in a blocking buffer $(50 \mathrm{mM}$ Tris $\mathrm{pH} 7.4,150 \mathrm{mM} \mathrm{NaCl}, 0.25 \%(\mathrm{w} / \mathrm{v})$ gelatine, and $0.5 \%$ triton $\mathrm{X}-100$ ) at room temperature for $15 \mathrm{~min}$, and afterwards with primary antibodies (Supplementary Table 2) in blocking buffer overnight at $4{ }^{\circ} \mathrm{C}$. Coverslips were washed with PBS and incubated with secondary antibodies (Supplementary Table 2) and Hoechst 33528 (1:1000, Thermo Fisher Scientific, H3569) in blocking buffer at room temperature for $1 \mathrm{~h}$. After washing steps with PBS, the coverslips were mounted on microscopy slides with Mowiol (0.1 M tris- $\mathrm{HCl}$ pH 8.5, 25\% glycerol, 10\% Mowiol (Merck Millipore, 81381). The samples were imaged using a Zeiss Axioscope A1 microscope with a $40 \times$ objective.

Generation of organotypic brain slices. For the generation of organotypic brain slices, the protocol of Pencheva et al., 2017 was adapted ${ }^{31}$. Postnatal day 15-17 C57BL6J pups were decapitated, the brains were dissected and captured in ice-cold artificial cerebrospinal fluid (aCSF, pH7.2: $10 \mathrm{mM}$ Hepes, $21 \mathrm{mM} \mathrm{NaHCO}$, $1.2 \mathrm{mM} \mathrm{NaH}_{2} \mathrm{PO}_{4}, 2.5 \mathrm{mM} \mathrm{KCl}, 2 \mathrm{mM} \mathrm{MgCl}_{2}, 2 \mathrm{mM} \mathrm{CaCl}_{2}, 5 \mathrm{mM}$ D-glucose, $250 \mathrm{mM}$ glycerol in milliQ). The brains were transferred to a petri dish and cerebellum and olfactory bulbs were removed. The cerebrum was glued to the vibratome cutting stage using a drop of Loctite 401 glue (Henkel Adhesives) with the rostral part facing upwards. The vibratome cutting stage was mounted on a VT1000S vibratome (Leica Biosystems, 1404723512) and tissue was fully submerged in carbonated ice-cold aCSF. Coronal brain slices of $350 \mu \mathrm{m}$ were cut with a speed of $0.1 \mathrm{~mm} / \mathrm{s}$ and a frequency of $7 \mathrm{~Hz}$. Slices with visible lateral ventricles were transferred to $1.0-\mu \mathrm{m}$ porous membrane inserts (Corning, 353102) in a 6-well plate with slicing medium [DMEM:F12 (Gibco, 11320), 1\% L-Glutamine (Gibco, 25030123), $5 \mathrm{mM}$ HEPES, $21 \mathrm{mM} \mathrm{NaHCO}_{3}$ and 1\% pen/strep (Gibco, 15140122], with a maximum of 4 slices per transwell insert. Residual aCSF was removed from the inserts, the brain slices were washed with PBS and the transwells were transferred to $1.5 \mathrm{~mL}$ recovery medium (DMEM:F12, 25\% FBS, $1 \%$ L-Glutamine, $5 \mathrm{mM}$ HEPES, $21 \mathrm{mM} \mathrm{NaHCO}_{3}$ and $1 \% \mathrm{P} / \mathrm{S}$ ) below the transwells, allowing the slices to be cultured at the air-liquid interface. The slices were cultured at $37^{\circ} \mathrm{C}$ in a humidified incubator with $5 \%$ $\mathrm{CO}_{2}$ overnight. The next day, the transwells were dipped twice in PBS and transferred to a 6-well plate containing NSC medium (DMEM:F12-GlutaMAX, 1\% pen/strep, 10 ng/mL EGF (Peprotech, AF-100-15-A), 10 ng/mL FGF (Peptrotech, AF-100-18B) before injection of cells.

Organotypic brain slice invasion assay. Cells were counted using the Countess 3 FL Automated Cell Counter (Thermo Fischer Scientific, AMQAF2000) and suspensions of $2.5 \times 10^{5} \mathrm{cells} / \mu \mathrm{l}$ were prepared. For the mixed cell injections, H2B-mNeonGreen expressing cells were mixed at a 1:1 ratio with $\mathrm{H} 2 \mathrm{~B}$-mCherry expressing internal control cells. For the CRISPR-Cas9 modulated cells, CTL-1-H2B-mCherry was used as an internal control (I-CTL1) for the CRISPR-set-A clones(CTL 1 and 2, GFAP $\delta$-KO 1 and 2, GFAPa-KO 1 and 2), and CTL-3-H2B-mCherry was used as an internal control (I-CTL2) for the CRISPR- set-B clones (CTL 3 and 4, GFAP $\delta$-KO 3 and 4, GFAPa-KO 3 and 4). For the shRNA modulated cells, NTC-H2B-mCherry was used as an internal control. A Hamilton $0.5 \mu \mathrm{L}$ syringe model $7000.5 \mathrm{KH}$ (Hamilton, 86250) was assembled on a Narishige micromanipulator model MM-3 (Narishige group) and was placed on the magnetic board of a Leica MS5 dissection microscope (Leica Biosystems), using a Narishige GJ-8 magnetic stand (Narishige group). The syringe was rinsed with acetone, $70 \%$ ethanol, and PBS before use. Before injection, the cell suspension was mixed and $0.5 \mu \mathrm{L}$ was taken up by the syringe. The needle was placed into the lateral ventricle of the brain slice by moving $50 \mu \mathrm{m}$ into the tissue and $40 \mu \mathrm{m}$ out. The cell suspension was slowly injected into the lateral ventricle of the mouse brain 
tissue, filling up the lateral ventricle without overflowing on the tissue. The medium of the organotypic brain slices was replaced every 2-3 days. One week after injection of the cells, the brain slices were washed with PBS and fixed in $4 \%$ PFA in PBS at $4{ }^{\circ} \mathrm{C}$ overnight.

Whole-mount immunostaining and RapiClear clearing. For whole-mount immunostaining of the organotypic brain slices, we used the method described in Belle et al. ${ }^{67}$. The porous membrane surrounding the brain tissue was cut out and transferred to a 24 -well dish. The tissue was permeabilised in $2 \%$ Triton-X-100 (Roche, 40319421) in PBS and subsequently incubated in PBSGT blocking solution (0.2\% gelatin, 0.5\% Triton$\mathrm{X}-100,0.01 \%$ thimerosal or $0.2 \%$ sodium azide in $1 \times \mathrm{PBS}$ ), for a minimum of $4 \mathrm{~h}$ at room temperature. Primary antibodies were diluted in PBSGT $+0.1 \%$ saponin, $300 \mu \mathrm{L}$ was added to the tissue and incubated on a horizontal shaker $(70 \mathrm{rpm})$ at $37^{\circ} \mathrm{C}$ for 3 days. Tissue injected with mixed $\mathrm{H} 2 \mathrm{~B}$-mNeonGreen/H2B-mCherry cells were incubated with rabbit anti-laminin antibodies (1:1000, Supplementary Table 2). The empty wells were filled with PBS to avoid evaporation of the primary antibody mix. The tissue was washed 6 times in PBS with $0.25 \%$ Triton-X-100 for one hour. Secondary antibodies were diluted in PBSGT $+0.1 \%$ saponin and the mix was spun down to precipitate aggregates. $300 \mu \mathrm{L}$ was added to the tissue and incubated on a horizontal shaker (70 rpm) at $37^{\circ} \mathrm{C}$ for $24 \mathrm{~h}$. The tissue was washed 6 times in PBS with $0.25 \%$ Triton-X-100 for one hour. For tissue clearing of the organotypic brain slices, we selected the RapiClear protocol developed by SunJin Lab, as this protocol does not lead to tissue shrinkage and the protocol is non-laborious ${ }^{32,68}$. For tissue clearing, the brain slices were transferred to iSpacers (SunJin Lab Co., \#IS002) mounted on microscope slides and $300 \mu \mathrm{L}$ of RapiClear 1.47 (SunJin Lab Co, \#RC147001) was added on the brain slices. The slices were cleared at $37^{\circ} \mathrm{C}$ on a horizontal shaker (30 rpm) for $45 \mathrm{~min}$, mounted with a coverslip in RapiClear 1.47, and sealed with transparent nail polish. The cleared brain slices were imaged using an LSM 880 (Zeiss) confocal microscope equipped with a 3-channel QUASAR Detection Unit (000000-2078-293). The entire population of injected cells was imaged with a $10 \times$ objective (N-Achroplan 10x, 420940-990-000) at $1.77 \mu \mathrm{m}$ pixel resolution Z-plane increments of $6.63 \mu \mathrm{m}$ and using image tiling. Smaller regions were imaged using a $20 \times$ objective (LD Plan-NEOFLUAR 20x, 421350 9970-000) at $0.42 \mu \mathrm{m}$ pixel resolution and $3.39 \mu \mathrm{m}$ Z-plane increments.

Quantification of cell invasion in ex vivo slices. Cell invasion in the organotypic brain slices was quantified in the confocal generated images using ImageJ (1.53c) and Imaris software (version 8.4). Upon blinding, images were excluded from analysis when errors had occurred during the injections of cells (overflowing of tissue, large populations of unhealthy looking cells). Using ImageJ software, image tiling was used to reconstruct the entire population of injected cells. The tiled z-stack consisted of an H2B-mNeonGreen and H2B-mCherry channel with the nuclei of the injected glioma cells and a laminin channel staining the mouse vasculature. In addition to staining the vasculature, laminin also gave rise to a diffuse staining at the location where tumour density was highest, as shown by H2B-mCherry/H2B-mNeonGreen signal. This staining of the ECM deposits generated by the tumour cells was used to draw a boundary between the tumour core and the mouse tissue (Supplementary Fig. 3) in the different z-planes. An additional channel was generated in which only the tumour core laminin staining was selected. The stitched images with additional laminin-channel were imported into the Imaris Software (version 8.4). The 'create surface' function was used to generate a 3D surface of the laminin tumour core signal (background subtraction, estimated diameter 17.8 , threshold $=2$, voxels $=1$ ). The 'create spots' function was used to generate individual spots $(11 \mu \mathrm{m}+$ PSF-elongation along the Z-axis) of the H2BmCherry nuclei using a standardised Quality threshold filter, adjusted so that cells in all z-planes were detected. Using the same function, the same number of H2B-mNeonGreen spots was generated. The 'distance transformation' function was used for the tumour core surface, generating a new channel where the intensity of the signal represented the distance from the 'outside surface object' or 'inside surface object'. The Imaris software was used to calculate for every $\mathrm{H} 2 \mathrm{~B}$-mCherry and $\mathrm{H} 2 \mathrm{~B}-\mathrm{mNeonGreen}$ nucleus the distance to the tumour core, using the 'intensity center' calculation within the "statistics" function. The excel file was exported and histograms of the distances (bin size $20 \mu \mathrm{m}$ ) were created using the NumPy package of the Python software ${ }^{69}$.

Tumour cell injection and cranial window implantation (CWI) surgery. Two clones per condition generated by two different sgRNA were used for the in vivo experiments: CTL 1 (CRISPR-set-A) and 3 (CRISPRset-B), GFAP $\delta$-KO 2 (CRISPR-set-A) and 3 (CRISPR-set-B), and GFAPa-KO 2 (CRISPR-set-A) and 4 (CRISPRset-B). Clones with the most extreme GFAP $\delta / a$ ratio were selected, except for the GFAPa-KO clone 3 as network collapses were observed in this line. Per injection, 100,000 U251-MG cells were resuspended in $3 \mu$ of PBS and injected the same day as the cranial window was implanted. CWI was performed as previously described ${ }^{70}$. In short, mice were sedated with $4 \%$ isoflurane inhalation for inducing anaesthesia and $1.5-2 \%$ during surgery. The hair from the back of the neck up to the eyes was shaved. Next, the mouse head was firmly fixed with ear bars in a stereotaxic device. Eye ointment was applied to prevent the animal's eyes from drying out. Next, the skin was cut circularly. After scraping the periosteum underneath to the edges of the skull, a circular groove of $5 \mathrm{~mm}$ diameter was drilled over the right parietal bone. After craniotomy, the dura mater was removed with a fine forceps. Next, tumour cells were injected stereotactically using a $10 \mu \mathrm{l}$ Hamilton syringe with a $2 \mathrm{pt}$ style needle in the middle of the craniotomy at a depth of $0.5 \mathrm{~mm}$. The exposed brain was sealed with silicone oil and a $6 \mathrm{~mm}$ coverslip glued on top. Dental acrylic cement (Vertex) was applied on the skull surface to cover the edge of the coverslip and a 3D printed plastic ring was glued around the coverslip to provide fixation to the microscope. A single dose of $100 \mu \mathrm{g} / \mathrm{kg}$ of buprenorphine (Temgesic, Indivior Europe Limited) was administered before the surgery and the day after surgery. In addition Rimadyl in water was administered $24 \mathrm{~h}$ before CIW implantation and for a total of $72 \mathrm{~h}$ (Zoetis). After surgery, the mice were provided food and water ad libitum. Mice were closely monitored twice per week. 
Intravital imaging. Mice were anaesthetised in an induction chamber with $4.0 \%$ isoflurane. Next, they were placed face-up in a custom-designed imaging box. A 3D printed imaging plate facilitated CWI fixation. Isoflurane was introduced through the facemask and ventilated by an outlet on the other side of the box. To study cell migration, time-lapse images of several positions of the tumour volume were acquired every $45 \mathrm{~min}$ for a maximum of $6 \mathrm{~h}$, during which the climate chamber surrounding the microscope was kept at $37^{\circ} \mathrm{C}$ and the mouse body temperature was monitored with a rectal thermometer. For each position, images of the complete $\mathrm{z}$ stack of the tumour were acquired, with a step size of $3 \mu \mathrm{m}$. Imaging was performed on an inverted Leica SP8 multiphoton microscope with a chameleon Vision-S (Coherent Inc., Santa Clare, CA, www.coherent.com). This microscope is equipped with a $25 \times$ (HCX IRAPO NA0.95 WD $2.5 \mathrm{~mm}$ ) water objective with four non-descanned detectors (NDDs). The NDDs detected the following wavelengths: NDD1 $<455 \mathrm{~nm}, \mathrm{NDD} 2455-505 \mathrm{~nm}$, NDD3 500-550 nm, NDD4 555-680 nm. H2B-mNeonGreen was excited with $944 \mathrm{~nm}$ and detected with NDD3. Scanning was performed in a bidirectional mode at $400 \mathrm{~Hz}$ and 12 bit, with a zoom of 1 , and $512 \times 512$ pixels.

Quantification of tumour density. Density was calculated at endpoint. A tumour was considered endpoint when approximately $50 \%$ or more of the cover slip of the imaging window was covered with tumour cells. All tumours included in the analysis were imaged between 13 and 35 days after the cranial window was implanted, except for one CTL1 which reached endpoint 72 days after window implantation due to a miss injection. To quantify the tumour density, we calculated the number of individual cells in the total tumour area. The quantification was done using ImageJ (U. S. NIH, Bethesda, Maryland, USA).

Tracking migration of tumour cells. All mice that were succesfully imaged for $6 \mathrm{~h}$ were included in the analysis. The analysis were done in a blinded manner. The tracking of migratory cells was done as previously described ${ }^{70}$. After imaging, acquired $\mathrm{z}$-stacks were corrected for $\mathrm{z}$ and xy shifts with Huygens Professional software program (version20.10). Up to 300 cells per mouse were tracked manually with an ImageJ plugin ("MTrackJ" Rasband, W.S., ImageJ, U. S. NIH, Bethesda, Maryland, USA). At the start of each movie, a random cell was selected. The XY position was determined over time and the displacement, speed and persistence for each cell were calculated by Excel (Microsoft).

The spatial average of all cell positions was used to measure the centre of mass displacement. For each border position, the centre of mass along the $\mathrm{Y}$-axis was measured by the 'Chemotaxis and Migration Tool. Calculation of the centre of mass $\left(\mathrm{M}_{\text {end }}\right)$. $\mathrm{i}$ = index of single cells, $\mathrm{n}=$ number of cells, $\mathrm{X}_{\mathrm{i} \text {,end }} \mathrm{Y}_{\mathrm{i} \text {,end }}=$ coordinates of the respective endpoint.

$$
M_{\text {end }}=\frac{1}{n} \sum_{i=1}^{n}\left(x_{i, \text { end }}, Y_{i, \text { end }}\right)
$$

Statistical analysis. The normality of data was tested using the Shapiro-Wilk test. For all normally distributed measurements, one-way ANOVA (when $>2$ means were compared) or two-way ANOVA followed by Tukey's multiple comparisons test were used to determine significance, set to $p<0.05$. For non-normally-distributed measurements, a Kruskal-Wallis test (when $>2$ means were compared) followed by Dunn's multiple comparisons test were used to determine significance. All $\mathrm{p}$ values were two-tailed. Levels of significance were set as follows: $n s>0.05,{ }^{\star} 0.05 \leq \mathrm{p}>0.01,{ }^{* *} 0.01 \leq \mathrm{p}>0.001,{ }^{\star * *} 0.001 \leq \mathrm{p}>0.0001,{ }^{* * *} \mathrm{p} \leq 0.0001$. Error bars are presented as mean \pm S.E.M. All statistical analyses were performed using GraphPad Prism software (version 9.1.2, GraphPad Software, USA).

\section{Data availability}

All data generated and analysed in this study are included in the manuscript and its supplementary files.

Received: 19 October 2021; Accepted: 16 December 2021

Published online: 10 January 2022

\section{References}

1. Ho, V. K. Y. et al. Changing incidence and improved survival of gliomas. Eur. J. Cancer 50, 2309-2318 (2014).

2. Bellail, A. C., Hunter, S. B., Brat, D. J., Tan, C. \& Van Meir, E. G. Microregional extracellular matrix heterogeneity in brain modulates glioma cell invasion. Int. J. Biochem. Cell Biol. 36, 1046-1069 (2004).

3. Hatoum, A., Mohammed, R. \& Zakieh, O. The unique invasiveness of glioblastoma and possible drug targets on extracellular matrix. Cancer Manag. Res. 11, 1843-1855 (2019).

4. Birbrair, A. 2017_Book_StemCellMicroenvironmentsAndBe. (2017).

5. Moeton, M. et al. Silencing GFAP isoforms in astrocytoma cells disturbs laminin-dependent motility and cell adhesion. FASEB J. 28, 2942-2954 (2014).

6. Stassen, O. M. J. A. et al. GFAPS/GFAPa ratio directs astrocytoma gene expression towards a more malignant profile. Oncotarget 8, 88104-88121 (2017).

7. van Bodegraven, E. J. et al. GFAP alternative splicing regulates glioma cell-ECM interaction in a DUSP4-dependent manner. FASEB J. 33, 12941-12959 (2019).

8. Leduc, C. \& Etienne-Manneville, S. Intermediate filaments in cell migration and invasion: The unusual suspects. Curr. Opin. Cell Biol. 32, 102-112 (2015)

9. Peter, A. \& Stick, R. Evolutionary aspects in intermediate filament proteins. Curr. Opin. Cell Biol. 32, 48-55 (2015).

10. Zhang, Y. \& Weinberg, R. A. Epithelial-to-mesenchymal transition in cancer: Complexity and opportunities EMT: A naturally occurring transdifferentia-tion program. Front. Med. 12, 1-13 (2018). 
11. Thiery, J. P., Acloque, H., Huang, R. Y. J. \& Nieto, M. A. Epithelial-mesenchymal transitions in development and disease. Cell 139, $871-890$ (2009).

12. Mendez, M. G., Kojima, S.-I. \& Goldman, R. D. Vimentin induces changes in cell shape, motility, and adhesion during the epithelial to mesenchymal transition. FASEB J. 24, 1838-1851 (2010).

13. Sharma, P., Alsharif, S., Fallatah, A. \& Chung, B. M. Intermediate filaments as effectors of cancer development and metastasis: A focus on keratins, vimentin, and nestin. Cells 8, 497 (2019).

14. Cheung, K. J., Gabrielson, E., Werb, Z. \& Ewald, A. J. Collective invasion in breast cancer requires a conserved basal epithelial program. Cell 155, 1639-1651 (2013).

15. Duffy, P. E. \& Rapport, M. M. The relationship of glial fibrillary acidic protein to the shape, motility, and differentiation of human astrocytoma cells. Exp. Cell Res. 139, 145-157 (1982).

16. Skalli, O. et al. Astrocytoma grade IV (glioblastoma multiforme) displays 3 subtypes with unique expression profiles of intermediate filament proteins. Hum. Pathol. 44, 2081-2088 (2013).

17. Leduc, C. \& Manneville, S. E. Regulation of microtubule-associated motors drives intermediate filament network polarization. J. Cell Biol. 216, 1689-1704 (2017).

18. Blechingberg, J., Lykke-andersen, S., Jensen, T. H., Jørgensen, A. L. \& Nielsen, A. L. Regulatory mechanisms for $3^{\prime}$-end alternative splicing and polyadenylation of the Glial Fibrillary Acidic Protein, GFAP, transcript. Nucleic Acids Res. 35, 7636-7650 (2007).

19. Nielsen, A. L. et al. A new splice variant of glial fibrillary acidic protein, GFAP epsilon, interacts with the presenilin proteins. J. Biol. Chem. 277, 29983-29991 (2002).

20. Moeton, M. et al. GFAP isoforms control intermediate filament network dynamics, cell morphology, and focal adhesions. Cell. Mol. Life Sci. 73, 4101-4120 (2016).

21. Nielsen, A. L. \& Jørgensen, A. L. Self-assembly of the cytoskeletal glial fibrillary acidic protein is inhibited by an isoform-specific C terminus. J. Biol. Chem. 279, 41537-41545 (2004).

22. van den Berge, S. A. et al. Longterm quiescent cells in the aged human subventricular neurogenic system specifically express GFAP- $\delta$. Aging Cell 9, 313-326 (2010).

23. Roelofs, R. F. et al. Adult human subventricular, subgranular, and subpial zones contain astrocytes with a specialized intermediate filament cytoskeleton. Glia 52, 289-300 (2005).

24. Blechingberg, J. et al. Identification and characterization of GFAPj, a novel glial fibrillary acidic protein isoform. Am. J. Trop. Med. Hyg. 51, 523-532 (1994).

25. Andreiuolo, F. et al. GFAP $\delta$ immunostaining improves visualization of normal and pathologic astrocytic heterogeneity. Neuropathology 29, 31-39 (2009).

26. Heo, D. H. et al. A histopathological diagnostic marker for human spinal astrocytoma: Expression of glial fibrillary acidic protein- $\delta$. J. Neurooncol. 108, 45-52 (2012).

27. Brehar, F. M., Arsene, D., Brinduse, L. A. \& Gorgan, M. R. Immunohistochemical analysis of GFAP-?? and nestin in cerebral astrocytomas. Brain Tumor Pathol. 32, 90-98 (2015).

28. Choi, K.-C., Kwak, S.-E., Kim, J.-E., Sheen, S. H. \& Kang, T.-C. Enhanced glial fibrillary acidic protein- $\delta$ expression in human astrocytic tumor. Neurosci. Lett. 463, 182-187 (2009).

29. van Bodegraven, E. J., van Asperen, J. V., Robe, P. A. J. \& Hol, E. M. Importance of GFAP isoform-specific analyses in astrocytoma. Glia 67, 1417-1433 (2019).

30. Perng, M.-D. et al. Glial fibrillary acidic protein filaments can tolerate the incorporation of assembly-compromised GFAP- $\delta$, but with consequences for filament organization and aB-crystallin association. Mol. Biol. Cell 82, 327-331 (2009).

31. Pencheva, N. et al. Identification of a druggable pathway controlling glioblastoma invasiveness. Cell Rep. 20, 48-60 (2017).

32. Bekkouche, B. M. B., Fritz, H. K. M., Rigosi, E. \& O'Carroll, D. C. Comparison of transparency and shrinkage during clearing of insect brains using media with tunable refractive index. Front. Neuroanat. 14, 1-19 (2020).

33. Margarido, A. S., Bornes, L., Vennin, C. \& van Rheenen, J. Cellular plasticity during metastasis: New insights provided by intravital microscopy. Cold Spring Harb. Perspect. Med. 10, 1-21 (2020).

34. Alieva, M. et al. Intravital imaging of glioma border morphology reveals distinctive cellular dynamics and contribution to tumor cell invasion. Sci. Rep. 9, 1-11 (2019).

35. McGregor, A. L., Hsia, C. R. \& Lammerding, J. Squish and squeeze-The nucleus as a physical barrier during migration in confined environments. Curr. Opin. Cell Biol. 40, 32-40 (2016).

36. Wolf, K. et al. Physical limits of cell migration: Control by ECM space and nuclear deformation and tuning by proteolysis and traction force. J. Cell Biol. 201, 1069-1084 (2013).

37. Ivkovic, S. et al. Direct inhibition of myosin II effectively blocks glioma invasion in the presence of multiple motogens. Mol. Biol. Cell 23, 533-542 (2012).

38. De Pascalis, C. et al. Intermediate filaments control collective migration by restricting traction forces and sustaining cell-cell contacts. J. Cell Biol. https://doi.org/10.1083/jcb.201801162 (2018).

39. Lepekhin, E. A. et al. Intermediate filaments regulate astrocyte motility. J. Neurochem. 79, 617-625 (2001).

40. Rutka, J. T. et al. Effects of antisense glial fibrillary acidic protein complementary DNA on the growth, invasion, and adhesion of human astrocytoma cells. Cancer Res. 54, 3267-3272 (1994).

41. Elobeid, A., Bongcam-Rudloff, E., Westermark, B. \& Nistér, M. Effects of inducible glial fibrillary acidic protein on glioma cell motility and proliferation. J. Neurosci. Res. 60, 245-256 (2000).

42. Gan, Z. et al. Vimentin intermediate filaments template microtubule networks to enhance persistence in cell polarity and directed migration. Cell Syst. 3, 252-263.e8 (2016).

43. Shabbir, S. H., Cleland, M. M., Goldman, R. D. \& Mrksich, M. Geometric control of vimentin intermediate filaments. Biomaterials 35, 1359-1366 (2014).

44. Schaedel, L., Lorenz, C., Schepers, A. V., Klumpp, S. \& Köster, S. Microtubules by direct interactions. Nat. Commun. https://doi. org/10.1038/s41467-021-23523-z (2021).

45. Petrie, R. J., Doyle, A. D. \& Yamada, K. M. Random versus directionally persistent cell migration. Nat. Rev. Mol. Cell Biol. 10, 538-549 (2009).

46. Trepat, X., Chen, Z. \& Jacobson, K. Cell migration. Compr. Physiol. 2, 2369-2392 (2012).

47. Forsyth, P. A. et al. Gelatinase-A (MMP-2), gelatinase-B (MMP-9) and membrane type matrix metalloproteinase-1 (MT1-MMP) are involved in different aspects of the pathophysiology of malignant gliomas. Br. J. Cancer 79, 1828-1835 (1999).

48. Broekman, M. L. et al. Multidimensional communication in the microenvirons of glioblastoma. Nat. Rev. Neurol. https://doi.org/ 10.1038/s41582-018-0025-8 (2018).

49. Markovic, D. S., Glass, R., Synowitz, M., Van Rooijen, N. \& Kettenmann, H. Microglia stimulate the invasiveness of glioma cells by increasing the activity of metalloprotease-2. J. Neuropathol. Exp. Neurol. 64, 754-762 (2005).

50. Chen, H. et al. Immune response in glioma's microenvironment.Innovative surgical sciences 5, 115-125 (2020)

51. Patel, A. P. et al. Single-cell RNA-seq highlights intratumoral heterogeneity in primary glioblastoma. Science (80-). 344, 1396-1401 (2014).

52. Szerlip, N. J. et al. Intratumoral heterogeneity of receptor tyrosine kinases EGFR and PDGFRA amplification in glioblastoma defines subpopulations with distinct growth factor response. Proc. Natl. Acad. Sci. U. S. A. 109, 3041-3046 (2012). 
53. Meyer, M. et al. Single cell-derived clonal analysis of human glioblastoma links functional and genomic heterogeneity. Proc. Natl. Acad. Sci. U. S. A. 112, 851-856 (2015).

54. Zeng, W. et al. Patient-derived xenografts of different grade gliomas retain the heterogeneous histological and genetic features of human gliomas. Cancer Cell Int. 20, 1-12 (2020).

55. Kahles, A. et al. Comprehensive analysis of alternative splicing across tumors from 8,705 patients. Cancer Cell 34, 211-224.e6 (2018).

56. Fuentes-Fayos, A. C. et al. Splicing machinery dysregulation drives glioblastoma development/aggressiveness: Oncogenic role of SRSF3. Brain 143, 3273-3293 (2020).

57. Voss, D. M., Sloan, A., Spina, R., Ames, H. M. \& Bar, E. E. The alternative splicing factor, MBNL1, inhibits glioblastoma tumor initiation and progression by reducing hypoxia-induced stemness. Cancer Res. 80, 4681-4692 (2020).

58. Gérard, M. et al. Hypoxia imaging and adaptive radiotherapy: A state-of-the-art approach in the management of glioma. Front. Med. 6, 117 (2019).

59. Jensen, R. L. et al. Preoperative dynamic contrast-enhanced MRI correlates with molecular markers of hypoxia and vascularity in specific areas of intratumoral microenvironment and is predictive of patient outcome. Neuro. Oncol. 16, 280-291 (2014).

60. Paz, I., Akerman, M., Dror, I., Kosti, I. \& Mandel-Gutfreund, Y. SFmap: A web server for motif analysis and prediction of splicing factor binding sites. Nucleic Acids Res. 38, 1-5 (2010).

61. Sun, W. et al. TSVdb: A web-tool for TCGA splicing variants analysis. BMC Genom. 19, 1-7 (2018).

62. Ran, F. A. et al. Genome engineering using the CRISPR-Cas9 system. Nat. Protoc. 8, 2281-2308 (2013).

63. Concordet, J. P. \& Haeussler, M. CRISPOR: Intuitive guide selection for CRISPR/Cas9 genome editing experiments and screens. Nucleic Acids Res. 46, W242-W245 (2018).

64. Doench, J. G. et al. Optimized sgRNA design to maximize activity and minimize off-target effects of CRISPR-Cas9. Nat. Biotechnol. 34, 184-191 (2016).

65. Root, D. E., Hacohen, N., Hahn, W. C., Lander, E. S. \& Sabatini, D. M. Genome-scale loss-of-function screening with a lentiviral RNAi library. Nat. Methods 3, 715-719 (2006).

66. Drost, J. et al. Sequential cancer mutations in cultured human intestinal stem cells. Nature 521, 43-47 (2015).

67. Belle, M. et al. A simple method for 3D analysis of immunolabeled axonal tracts in a transparent nervous system. Cell Rep. 9, 1191-1201 (2014).

68. www.sunjinlab.com. Mouse Brain Quick Guide. Accessed 4 Oct 2017.

69. Harris, C. R. et al. Array programming with NumPy. Nature 585, 357-362 (2020).

70. Alieva, M. et al. Preventing inflammation inhibits biopsy-mediated changes in tumor cell behavior. Sci. Rep. 7, 7529 (2017).

\title{
Acknowledgements
}

The results shown here are partly based on data generated by The Cancer Genome Atlas Research Network (https://www.cancer.gov/tcga). The authors thank all members of the Hol and van Rheenen labs for thoughtful discussion. This study was funded by the Dutch Cancer Society [KWF 101123, R.U.C, J.v.A, J.v.R, E.H.], a HFSP fellowship (C.V.), the Portuguese Foundation for Science and Technology (FCT, GABBA program-PD/ BD/105748/2014, A.S.M.), the T and P Bohnenn Foundation (P.R) and the Josef Steiner Foundation (J.v.R).

\section{Author contributions}

R.U.C and J.A. performed conceptualization, data collection, formal analysis, methodology, and wrote the original draft. C.V. performed conceptualization, methodology, supervision, and reviewed and edited the manuscript. J.S. performed data collection, methodology, and reviewed and edited the manuscript. E.B. performed conceptualization, methodology, and reviewed and edited the manuscript. A.S.M. performed methodology, and reviewed and edited the manuscript. P.R. performed conceptualization, methodology, supervision, and reviewed and edited the manuscript. J.R. performed conceptualization, funding acquisition, project administration, methodology, supervision, and reviewed and edited the manuscript. E.H. performed conceptualization, funding acquisition, project administration, methodology, supervision, and reviewed and edited the manuscript.

\section{Competing interests}

The authors declare no competing interests.

\section{Additional information}

Supplementary Information The online version contains supplementary material available at https://doi.org/ 10.1038/s41598-021-04127-5.

Correspondence and requests for materials should be addressed to J.R. or E.M.H.

Reprints and permissions information is available at www.nature.com/reprints.

Publisher's note Springer Nature remains neutral with regard to jurisdictional claims in published maps and institutional affiliations.

\begin{abstract}
Open Access This article is licensed under a Creative Commons Attribution 4.0 International Lecense, which permits use, sharing, adaptation, distribution and reproduction in any medium or
format, as long as you give appropriate credit to the original author(s) and the source, provide a link to the Creative Commons licence, and indicate if changes were made. The images or other third party material in this article are included in the article's Creative Commons licence, unless indicated otherwise in a credit line to the material. If material is not included in the article's Creative Commons licence and your intended use is not permitted by statutory regulation or exceeds the permitted use, you will need to obtain permission directly from the copyright holder. To view a copy of this licence, visit http://creativecommons.org/licenses/by/4.0/.
\end{abstract}

(C) The Author(s) 2022 